\title{
Thermodynamics and its correlation with dynamics in a mean-field model and pinned systems: A comparative study using two different methods of entropy calculation
}

Cite as: J. Chem. Phys. 156, 014503 (2022); https://doi.org/10.1063/5.0065668

Submitted: 04 August 2021 - Accepted: 14 December 2021 • Accepted Manuscript Online: 15 December 2021 • Published Online: 04 January 2022

(D) Ujjwal Kumar Nandi, (D) Palak Patel, (D) Mohd Moid, et al.

\section{COLLECTIONS}

Paper published as part of the special topic on Slow Dynamics
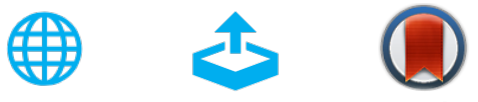

\section{ARTICLES YOU MAY BE INTERESTED IN}

\section{Perturbation-adapted perturbation theory}

The Journal of Chemical Physics 156, 011101 (2022); https://doi.org/10.1063/5.0079853

On the thermodynamics of curved interfaces and the nucleation of hard spheres in a finite system

The Journal of Chemical Physics 156, 014505 (2022); https://doi.org/10.1063/5.0072175

Accurate scaling functions of the scaled Schrödinger equation

The Journal of Chemical Physics 156, 014113 (2022); https://doi.org/10.1063/5.0077495

\section{Chemical Physics Reviews}

\section{First Articles Now Onlinel $=$ READ NOW »»}




\title{
Thermodynamics and its correlation
}

\section{with dynamics in a mean-field model and pinned systems: A comparative study using two different methods of entropy calculation}

\author{
Cite as: J. Chem. Phys. 156, 014503 (2022); doi: 10.1063/5.0065668 \\ Submitted: 4 August 2021 - Accepted: 14 December 2021 • \\ Published Online: 4 January 2022
}

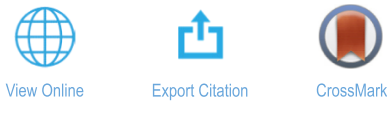

\begin{abstract}
Ujjwal Kumar Nandi, ${ }^{1,2}$ (D) Palak Patel, ${ }^{1,2}$ (D) Mohd Moid, ${ }^{3}$ (D) Manoj Kumar Nandi, ${ }^{4}$ (D) Shiladitya Sengupta, Smarajit Karmakar, ${ }^{6}$ (D) Prabal K. Maiti, ${ }^{3}$ (D) Chandan Dasgupta, ${ }^{3,7}$ (D) and Sarika Maitra Bhattacharyya ${ }^{1,2, a)}$ (D)

AFFILIATIONS

${ }^{1}$ Polymer Science and Engineering Division, CSIR-National Chemical Laboratory, Pune 411008, India

${ }^{2}$ Academy of Scientific and Innovative Research (AcSIR), Ghaziabad 201002, India

${ }^{3}$ Centre for Condensed Matter Theory, Department of Physics, Indian Institute of Science, Bangalore 560012, India

“Department of Engineering, University of Campania "Luigi Vanvitelli", 81031 Aversa (CE), Italy

${ }^{5}$ Department of Physics, Indian Institute of Technology, Roorkee 247667, India

${ }^{6}$ Centre for Interdisciplinary Sciences, Tata Institute of Fundamental Research, 36/P, Gopanpally Village, Serilingampally Mandal, RR District, Hyderabad 500019, India

${ }^{7}$ International Centre for Theoretical Sciences, Tata Institute of Fundamental Research, Bengaluru 560089, India
\end{abstract}

Note: This paper is part of the JCP Special Topic on Slow Dynamics.

a) Author to whom correspondence should be addressed: mb.sarika@ncl.res.in

\begin{abstract}
A recent study introduced a novel mean-field model system where each particle over and above the interaction with its regular neighbors interacts with $k$ extra pseudo-neighbors. Here, we present an extensive study of thermodynamics and its correlation with the dynamics of this system. We surprisingly find that the well-known thermodynamic integration (TI) method of calculating the entropy provides unphysical results. It predicts vanishing of the configurational entropy at temperatures close to the onset temperature of the system and negative values of the configurational entropy at lower temperatures. Interestingly, well below the temperature at which the configurational entropy vanishes, both the collective and the single-particle dynamics of the system show complete relaxation. Negative values of the configurational entropy are unphysical, and complete relaxation when the configurational entropy is zero violates the prediction of the random first-order transition theory (RFOT). However, the entropy calculated using the two-phase thermodynamics (2PT) method remains positive at all temperatures for which we can equilibrate the system, and its values are consistent with RFOT predictions. We find that with an increase in $k$, the difference in the entropy computed using the two methods increases. A similar effect is also observed for a system where a randomly selected fraction of the particles are pinned in their positions in the equilibrated liquid. We show that the difference in entropy calculated via the 2PT and TI methods increases with pinning density.
\end{abstract}

Published under an exclusive license by AIP Publishing. https://doi.org/10.1063/5.0065668

\section{INTRODUCTION}

The slowing down of the dynamics in supercooled liquids and its correlation with the thermodynamics of the system have been topics of intense research. There are several characteristic temperatures where both thermodynamic and dynamic properties of the system change in a significant manner. At the onset temperature $\left(T_{\text {onset }}\right)$, the relaxation dynamics of the system start to differ from those of a typical liquid because, due to the lowering of the temperature, the system begins to explore the underlying free energy 
landscape. ${ }^{1}$ This onset temperature can also be identified as the temperature where the pair part of the excess entropy becomes less than the total excess entropy of the system. ${ }^{2,3}$ Below $T_{\text {onset }}$, the temperature dependence of the dynamics can be described reasonably well by the so-called mode-coupling theory (MCT), which predicts a power-law divergence of the relaxation times at a dynamic transition temperature $T_{c} \cdot{ }^{4}$ However, experimental and numerical studies found ${ }^{5-10}$ that the relaxation time does not diverge at $T_{c}$ as predicted by the MCT but instead shows a smooth crossover to weaker temperature dependence. This crossover scenario is consistent with the predictions of the so-called random first-order transition (RFOT) theory, ${ }^{11,12}$ and it has been related to the properties of the underlying potential energy landscape. ${ }^{13}$

According to the RFOT theory and the phenomenological Adam-Gibbs (AG) theory, ${ }^{14}$ the low temperature dynamics of a supercooled liquid is controlled by its configurational entropy $\left(S_{c}\right)$, which measures the number of possible distinct states accessible to the system. The AG theory predicts the following relationship between the $\alpha$ relaxation time $(\tau)$ and the configurational entropy $\left(S_{c}\right): \tau=\tau_{0} \exp \left(A / T S_{c}\right)$, where $\tau_{0}$ is a microscopic timescale and $A$ is a system-dependent constant. Thus, according to the AG theory, the temperature $T_{0}$ where the relaxation time diverges is the same as the Kauzmann temperature $T_{K}$ where the configurational entropy goes to zero. ${ }^{15}$ For a large number of systems, the AG relationship is found to hold. ${ }^{14,16-25}$ There has been a recent study that showed that it is the diffusion coefficient that follows the AG relationship for the widest temperature range. ${ }^{26}$

The validity of the AG theory in the form presented above has recently been challenged. ${ }^{16}$ It has been argued that according to the RFOT theory, the reduction in the configurational entropy is related to the growth of a static correlation length over which the activation happens, giving rise to the relaxation process. This theory predicts a generalized AG relation given by $\tau=\tau_{0} \exp \left(A / T S_{c}^{\alpha}\right)$, where $\alpha$ can be different from unity. It was further shown that the generalized AG relation holds ${ }^{16}$ both in experiments and in simulations. Note that even according to the generalized AG relationship, the relaxation timescale should diverge below $T=T_{K}$ when the configurational entropy vanishes. In addition, by definition, $S_{c}$ cannot be negative.

In a recent study, some of us have developed a novel generalization of glass-forming liquids where we can switch between a three-dimensional liquid and a fully connected mean-field system in a continuous manner. ${ }^{27}$ The parameter that is introduced to achieve this is $k$ added pseudoneighbors for each particle. The structure, dynamics, and dynamical heterogeneity of this model have been studied as a function of $k$. It was shown that the structure given by the radial distribution function ( $\mathrm{rdf}$ ) of the usual neighbors remains almost unchanged with $k$. However, the pseudoneighbors do contribute to the total rdf that shows a weaker modulation with distance, a typical mean-field like behavior. ${ }^{27,28}$ With an increase in $k$, the dynamics also slows down and the transition temperatures $\left(T_{0}, T_{c}, T_{\text {onset }}\right)$ move to higher values. The range over which a system follows the MCT power-law behavior becomes wider with an increase in $k$. The heterogeneity decreases with an increase in $k$. Thus, it was shown that with an increase in $k$, the system becomes more mean-field like.

The goal of the present work is to study the thermodynamic properties of this system and its correlation with the dynamics.
In order to do so, we employ the well-known thermodynamic integration (TI) method to calculate the total entropy and, hence, the configurational entropy of the system. ${ }^{29}$ We find that with an increase in $k$, the Kauzmann temperature becomes higher, which is similar to that found for $T_{0}$. However, for larger $k$ systems, we find that the configurational entropy vanishes at temperatures close to the onset temperature. On the contrary, both the self-part and the collective part of the dynamics show complete relaxation well below the temperature where the configurational entropy vanishes. Thus, we can apparently access states with negative configurational entropy. This is an unphysical result by definition. In addition, it also violates the RFOT prediction. Thus, in our opinion, this observation implies that the TI method of entropy calculation should be re-examined. We discuss the possible failure points of the TI method. However, at present, we do not know how to incorporate the corrections.

We, thus, employ a completely different method to calculate the entropy of the system, namely, the two-phase thermodynamics (2PT) method. It is a well-known method ${ }^{30,31}$ that has provided accurate entropy values over a wide range of thermodynamic state points for the Lennard-Jones (LJ) fluid and different water models. ${ }^{30,32}$ We first test this method for a standard Kob-Anderson (KA) model, which is the $k=0$ system in the mean-field model. We compare the entropy values obtained via the TI and the 2PT methods and find them close to each other. We then employ the 2PT method for different mean-field systems and compare the results with those obtained by the TI method. We find that with increasing $k$, the difference in entropy obtained by the two methods increases. The entropy calculated using the $2 \mathrm{PT}$ method does not produce any negative configurational entropy state points. We also find that it predicts a Kauzmann temperature, which is similar to the temperature where the $\alpha$ relaxation time diverges, thus validating the RFOT prediction. Incidentally, we find that in the range of temperature studied here, the standard AG relation holds.

Similar to the mean-field system, there has been some discussion of the dynamics not following the entropy and the breakdown of the AG relationship when the entropy was calculated using the TI method in another model, namely, randomly pinned systems. ${ }^{33-}$ Given the success of the 2PT method in describing the entropy of the mean-field system, we further employ it to calculate the entropy of the pinned system. We find that with the increase in the pinning density, the difference in entropy computed by the TI and the 2PT methods increases. We also show that in the temperature range studied, the pinned systems follow the AG relationship when the entropy is calculated via the 2PT method.

Although our simulations provide results at relatively high temperatures at which the systems can be equilibrated, they offer an opportunity to critically test theories of the glass transition. The TI method is an important standard method to estimate entropy reliably and easily, both in experiments and in simulations. The unphysical results produced by the TI method for the mean-field model is rather surprising. Given their importance, they should be critically examined. Thus, in the present study, our main focus is on understanding possible issues with entropy calculation in these models.

The rest of this paper is organized as follows: The system and simulation details are described in Sec. II. In Sec. III, we describe different methods for the calculation of entropy. In Secs. IV and V, 
we present the results of our analysis for the mean-field and pinned systems, respectively. In Sec. VI, we summarize the results and conclude.

\section{DETAILS OF SYSTEM AND SIMULATIONS}

We have studied two different families of models. One is a mean-field system and the other is a pinned system. For both the systems, we have used atomistic models, which are simulated with two-component mixtures of classical particles (larger " $A$ " and smaller "B" type), where particles of type $i$ interact with those of type $j$ with pair potential, $u\left(r_{i j}\right)$, where $r$ is the distance between the pair. $u\left(r_{i j}\right)$ is described by a shifted and truncated Lennard-Jones (LJ) potential, given by

$$
u\left(r_{i j}\right)= \begin{cases}u^{(L J)}\left(r_{i j} ; \sigma_{i j}, \epsilon_{i j}\right)-u^{(L J)}\left(r_{i j}^{(c)} ; \sigma_{i j}, \epsilon_{i j}\right), & r \leq r_{i j}^{(c)}, \\ 0, & r>r_{i j}^{(c)},\end{cases}
$$

where $u^{(L J)}\left(r_{i j} ; \sigma_{i j}, \epsilon_{i j}\right)=4 \epsilon_{i j}\left[\left(\frac{\sigma_{i j}}{r_{i j}}\right)^{12}-\left(\frac{\sigma_{i j}}{r_{i j}}\right)^{6}\right]$ and $r_{i j}^{(c)}=2.5 \sigma_{i j}$. We have used the Kob-Andersen model $^{8}$ and performed constant volume and constant temperature (Nosé-Hoover thermostat and velocity rescaling) molecular dynamics (NVTMD) simulation. We use $\sigma_{A A}$ and $\epsilon_{A A}$ as the units of length and energy, respectively, setting the Boltzmann constant $k_{B}=1$. We have used reduced time unit in terms of $\sqrt{\frac{m_{A} \sigma_{A A}^{2}}{\epsilon_{A A}}}$, and masses of both types of particles are taken to be the same $\left(m_{A}=m_{B}\right.$, set equal to unity). We have used $80 \%$ of A particles and $20 \%$ of B particles with the diameter $\sigma_{A A}=1.0$, $\sigma_{A B}=0.8$, and $\sigma_{B B}=0.88$. The interaction strengths between the particles are $\epsilon_{A A}=1.0, \epsilon_{A B}=1.5$, and $\epsilon_{B B}=0.5$.

\section{A. Mean-field system}

The mean-field system is given by $N$ particles that interact with each other via a standard short-range potential. In addition, each particle also interacts with "pseudoneighbors," i.e., particles that are not necessarily close in space. Hence, the total interaction potential of the system is given by

$$
\begin{aligned}
U_{\text {tot }}\left(r_{1}, \ldots, r_{N}\right) & =\sum_{i=1}^{N} \sum_{j>i}^{N} u\left(r_{i j}\right)+\frac{1}{2} \sum_{i=1}^{N} \sum_{j=1}^{k} u^{\text {pseudo }}\left(r_{i j}\right) \\
& =U+U_{k}^{\text {pseudo }} .
\end{aligned}
$$

The first term on the right-hand side is the regular interaction between particles, while the second term is the interaction each particle has with its pseudoneighbors. Here, we consider the case in which the regular interaction is described by using Eq. (1).

The interaction potential with the pseudoneighbors is modeled in terms of a modified shifted and truncated LJ potential,

$$
\begin{aligned}
u^{\text {pseudo }}\left(r_{i j}\right) & =u\left(r_{i j}-L_{i j}\right) \\
& =4 \epsilon_{i j}\left[\left(\frac{\sigma_{i j}}{r_{i j}-L_{i j}}\right)^{12}-\left(\frac{\sigma_{i j}}{r_{i j}-L_{i j}}\right)^{6}\right],
\end{aligned}
$$

where $L_{i j}$ is a random variable defined below. In our simulations, we impose the restriction that any two particles interact either via $u\left(r_{i j}\right)$ or via $u^{\text {pseudo }}\left(r_{i j}\right)$. This condition determines how the pseudoneighbors and the values $L_{i j}$ are chosen for a given configuration equilibrated with the potential $u$ : for each particle $i$, we select $k$ random numbers $L_{i j}$ in the range $r_{c} \leq L_{i j} \leq L_{\max }$, where $L_{\max } \leq L_{\mathrm{box}} / 2-r_{c}$, with $L_{\mathrm{box}}$ being the size of the simulation box. [The distribution of these random variables will be denoted by $\mathscr{P}\left(L_{i j}\right)$, and in the following, we will consider the case that the distribution is uniform.] Subsequently, we choose $k$ distinct particles $j$ with $r_{i j}>r_{c}$ and use the $L_{i j}$ to permanently fix the interaction between particles $i$ and $j$. This procedure thus makes sure that each particle $i$ interacts with not only the particles that are within the cutoff distance but also $k$ particles that can be far away. Note that once the particle $j$ is chosen as a pseudoneighbor of particle $i$, automatically particle $i$ becomes a pseudoneighbor of particle $j$. The system, as defined here, can then be simulated using standard simulation algorithms.

NVT molecular dynamics (MD) simulation is performed in a cubic box using the velocity rescaling method for $N=2744$ particles at $\rho=1.2\left(L_{\mathrm{box}}=13.1745\right)$ using a time integration step of $\Delta t=0.005$. For $L_{\max }$, we have taken 4.0 , slightly below the maximum value of 4.09. We have simulated four different systems with the number of pseudoneighbors, $k=0,4,12$, and 28 .

\section{B. Pinned system}

We also study another conceptually different family of glassforming models, namely, the pinned system in which a fraction of particles are artificially pinned at their positions in an equilibrated liquid. For the study of the pinned system, we use the Kob-Andersen 80:20 binary Lenard-Jones mixture. ${ }^{8}$ Details of this model are given in Sec. II. For creating the pinned system, the following pinning protocol is used. The pinned particles are chosen randomly from an equilibrium configuration of the system at the temperature of interest. ${ }^{33,35}$ NVT molecular dynamics simulation is performed in a cubic box using the Nose-Hoover thermostat where $\mathrm{N}=1000$ at $\rho=1.2\left(L_{b o x}=9.41036\right)$ using a time integration step of $\Delta t=0.005$, at three different pinning concentration $(c)$, i.e., $0.05,0.10$, and 0.15 . Production runs of pinned configurations are long enough to ensure that within the simulation time, the overlap correlation function $\mathrm{Q}(\mathrm{t})$ (defined in Sec. II C) decays to zero.

\section{Dynamics}

To analyze the dynamics, we consider the self-part of the overlap function,

$$
Q(t)=\frac{1}{N}\left\langle\sum_{i=1}^{N} \omega\left(\left|\mathbf{r}_{i}(t)-\mathbf{r}_{i}(0)\right|\right)\right\rangle
$$

where the function $\omega(x)$ is 1 if $0 \leq x \leq a$ and $\omega(x)=0$ otherwise. The parameter $a$ is chosen to be 0.3 , a value that is slightly larger than the size of the "cage" determined from the height of the plateau in the mean square displacement at intermediate times. ${ }^{8}$ Thus, the quantity $Q(t)$ measures whether or not at time $t$ a tagged particle is still inside the cage it occupied at $t=0$. 
To analyze the collective dynamics of the systems, we have used both the collective overlap function and the collective intermediate scattering function.

The collective overlap function is defined as follows:

$$
Q^{t o t}(t)=\frac{1}{N}\left\langle\sum_{i=1}^{N} \sum_{j=1}^{N} \omega\left(\left|\mathbf{r}_{i}(t)-\mathbf{r}_{j}(0)\right|\right)\right\rangle \text {. }
$$

The long time saturation value of $\mathrm{Q}^{\text {tot }}(\mathrm{t})$ is given by (using $\left.\mathrm{a}=0.3\right)^{3}$

$$
\lim _{t \rightarrow \infty} Q^{t o t}(t)=\frac{N}{V} \frac{4}{3} \pi a^{3}=0.135
$$

We have also calculated the intermediate scattering function $F(q, t)$. It is the collective density-density time correlation function in momentum space which provides information about the collective dynamics of the systems,

$$
F(q, t)=\frac{1}{N F(q, 0)}\left\langle\sum_{i=1}^{N} \sum_{j=1}^{N} \exp \left[-i \mathbf{q} \cdot\left(\mathbf{r}_{i}(t)-\mathbf{r}_{j}(0)\right)\right]\right\rangle .
$$

The relaxation time $(\tau)$ is calculated from the self-part of the overlap function when it decays to $1 /$ e. The rapid increase in relaxation time with decreasing temperature is a signature of glassy dynamics. This is often fitted to the Vogel-Fulcher-Tammann (VFT) equation,

$$
\tau(T)=\tau_{0} \exp \left[\frac{1}{K\left(\frac{T}{T_{0}}-1\right)}\right]
$$

Here, $\tau_{0}$ is a high-temperature relaxation time and $T_{0}$ is the so-called VFT temperature at which the relaxation time of the system is predicted to diverge. The parameter $K$ describes the curvature of the data in an Arrhenius plot and, hence, can be considered as a measure for the fragility of the glass-former.

\section{ENTROPY}

In this work, we have used two different well-known methods for the calculation of the total entropy $\left(S_{t o t}\right)$ of the system. Below, we provide brief sketches of the two methods, namely, the TI method ${ }^{29}$ and the $2 \mathrm{PT}$ method. ${ }^{30}$

\section{A. Thermodynamic integration (TI) method}

Below, we describe the different quantities required to calculate the entropy in the TI method. ${ }^{29}$

\section{Ideal gas entropy}

Ideal gas entropy is the entropy of a set of non-interacting particles. The ideal gas entropy per particle for a binary system at temperature $T$ is given by

$$
S_{\text {ideal }}=\frac{5}{2}-\ln (\rho)+\frac{3}{2} \ln \left(\frac{2 \pi T}{h^{2}}\right)+\frac{1}{N} \ln \frac{N !}{N_{A} ! N_{B} !},
$$

where $N=N_{A}+N_{B}$ is the total number of particles, $\rho$ is the density of the system, and $h$ is the Planck constant. $N_{A}$ and $N_{B}$ are the number of particles of types $\mathrm{A}$ and $\mathrm{B}$. The last term contributes to the mixing entropy.

However, if the particles are divided into $M$ distinguishable species such that $N=\sum_{i=1}^{M} N_{i}$, then the ideal gas entropy per particle can written as

$$
S_{\text {ideal }}^{d}=\frac{5}{2}-\ln (\rho)+\frac{3}{2} \ln \left(\frac{2 \pi T}{h^{2}}\right)+\frac{1}{N} \ln \frac{N !}{\Pi_{i=1}^{M} N_{i} !} .
$$

\section{Excess entropy and total entropy}

Excess entropy $\left(S_{e x}\right)$ estimates the loss of entropy due to interactions among the particles. It is always a negative quantity. $S_{e x}$ is calculated using the TI method where the integration can be done on the temperature path, ${ }^{33}$ in the temperature range $\infty$ to a target temperature $\left(T^{*}\right)$,

$$
S_{e x}\left(\beta^{*}\right)=\beta^{*}\langle U\rangle-\int_{0}^{\beta^{*}} d \beta\langle U\rangle
$$

Here, $\beta=\frac{1}{T}$. The total entropy of the system at a particular temperature is the sum of the ideal gas entropy and the excess entropy of the system at that particular temperature,

$$
S_{\text {tot }}=S_{\text {ideal }}+S_{\text {ex }}
$$

\section{B. Two-phase thermodynamics (2PT) method}

The 2PT method is another conventional method to compute the entropy of liquids. ${ }^{30,31}$ In the 2PT method, the thermodynamics quantities can be computed using the density of states (DOS) of the liquid. One can decompose the DOS of a liquid as a sum of solid-like and gas-like contributions. To compute the thermodynamic quantities, the phonons in the solid-like DOS are treated as non-interacting harmonic oscillators, as in the Debye model..$^{38}$ On the other hand, the gas-like DOS is described as a low-density hard-sphere fluid, which can be computed analytically. ${ }^{38}$ Using the $2 \mathrm{PT}$ description, Lin et al..$^{30,31}$ demonstrated that the thermodynamics quantities of the $\mathrm{LJ}$ fluid can be computed very accurately over a wide range of thermodynamics state points using a very short MD trajectory. In a later work, Lai et al. ${ }^{39}$ calculated the entropy of a binary fluid using the 2PT method. Here, we provide a brief overview of the decomposition of the DOS in the $2 \mathrm{PT}$ method. We refer the reader to the original papers ${ }^{30,31}$ for a full description.

The density of state function, $\mathrm{g}(v)$, can be computed from the mass-weighted atomic spectral densities, defined as ${ }^{30,31}$

$$
\mathrm{g}(v)=\frac{2}{T} \sum_{j=1}^{N} \sum_{l=1}^{3} m_{j} s_{j}^{l}(v),
$$

where $m_{j}$ is the mass of the $j$ th atom, $l$ denotes the direction in the Cartesian coordinates, and $s_{j}^{l}(v)$ are the atomic spectral densities, defined as

$$
s_{j}^{l}(v)=\lim _{\tau \rightarrow \infty} \frac{\left|\int_{-\tau}^{\tau} v_{j}^{l}(t) e^{-i 2 \pi v t} d t\right|^{2}}{2 \tau},
$$


where $v_{j}^{l}(t)$ denotes the velocity component of the $j$ th atom in the $l$ th direction. The atomic spectral density, $s_{j}^{l}(v)$, can be computed from the Fourier transform of the velocity auto-correlation function (VACF) $c_{j}^{l}(t)$

$$
s_{j}^{l}(v)=\lim _{\tau \rightarrow \infty} \int_{-\tau}^{\tau} c_{j}^{l}(t) e^{-i 2 \pi v t} d t
$$

where $c_{j}^{l}(t)$ is given by

$$
c_{j}^{l}(t)=\lim _{\tau \rightarrow \infty} \frac{1}{2 \tau} \int_{-\tau}^{\tau} v_{j}^{l}\left(t+t^{\prime}\right) v_{j}^{l}\left(t^{\prime}\right) d t^{\prime} .
$$

Thus, Eq. (15) can be rewritten as

$$
\mathrm{g}(v)=\frac{2}{T} \lim _{\tau \rightarrow \infty} \int_{-\tau}^{\tau} \sum_{j=1}^{N} \sum_{l=1}^{3} m_{j} c_{j}^{l}(t) e^{-i 2 \pi v t} d t
$$

As we mentioned above, $\mathrm{g}(v)$ can be decomposed into solidand gas-like components in the $2 \mathrm{PT}$ formalism. Based on the diffusivity of the system compared to hard-sphere gas at the same density, Lin et al. ${ }^{30}$ proposed a self-consistent fluidity factor, $f$, which decides the degrees of freedom shared in solid and gas components. The relationship between $f$ and dimensionless diffusivity, $\Delta$, can be derived (for the details of the derivation, readers are referred to Ref. 30) by

$$
2 \Delta^{-9 / 2} f^{15 / 2}-6 \Delta^{-3} f^{5}-\Delta^{-3 / 2} f^{7 / 2}+6 \Delta^{-3 / 2} f^{5 / 2}+2 f-2=0 .
$$

The dimensionless diffusivity constant, $\Delta$, depends on the material properties,

$$
\Delta\left(T, \rho, m, \mathrm{~g}_{0}\right)=\frac{2 \mathrm{~g}_{0}}{9 N}\left(\frac{6}{\pi}\right)^{2 / 3}\left(\frac{\pi T}{m}\right)^{1 / 2} \rho^{1 / 3},
$$

where $g_{0}=g(0)$ is the DOS of the system at zero-frequency and $\rho$ is the number density. Using $f$ obtained from Eqs. (20) and (21), the DOS in the gas-like diffusive component can be obtained using a hard-sphere diffusive model,

$$
g^{g}(v)=\frac{g_{0}}{1+\left[\frac{\pi g_{0} v}{6 f N}\right]^{2}} .
$$

Given the DOS in the gas-like component, one can compute the solid-like DOS, $\mathrm{g}^{s}(v)$, using the following equation:

$$
\mathrm{g}(v)=\mathrm{g}^{\mathrm{g}}(v)+\mathrm{g}^{s}(v)
$$

Once the decomposition of the DOS has been done, any thermodynamic quantity, $A$, can be computed using the corresponding weight functions,

$$
A=\beta^{-1}\left[\int_{0}^{\infty} \mathrm{g}^{g}(v) W_{A}^{g} d v+\int_{0}^{\infty} \mathrm{g}^{s}(v) W_{A}^{s} d v\right] .
$$

The weight functions for the entropy in the solid- $\left(\mathrm{W}_{S}^{s}\right)$ and the gas-like $\left(\mathrm{W}_{S}^{g}\right)$ components are defined as

$$
\mathrm{W}_{S}^{s}(v)=\mathrm{W}_{S}^{H O}(v)=\frac{\beta \hbar v}{\exp (\beta \hbar v)-1}-\ln [1-\exp (-\beta \hbar v)]
$$

where $\beta=\frac{1}{T}$ and $\hbar=\frac{h}{2 \pi}$ and $\mathrm{h}$ is the Planck constant,

$$
\mathrm{W}_{S}^{g}(v)=\frac{1}{3} \frac{S^{H S}}{k}
$$

where $S^{H S}$ denotes the entropy of the hard-sphere system. Using Eqs. (25) and (26), the total entropy of the system can be written as

$$
S_{t o t}=S^{s}+S^{\mathrm{g}} .
$$

In this work, for the calculation of the entropy using the $2 \mathrm{PT}$ method, we have averaged over ten datasets where each dataset starts with a different configuration and velocity distribution. Each dataset contains fifty thousand frames of velocity with an interval of 0.005 time steps.

\section{Configurational entropy}

As discussed earlier, we can calculate the total entropy using both the TI and the 2PT methods. Thus, Eqs. (14) and (27) provide us with the same information although the routes of obtaining them are different.

In the supercooled liquid regime, the configurational space can be divided into inherent structure minima and vibrational motion around them. The logarithm of the number of these inherent structure minima gives the configurational entropy $\left(S_{c}\right)$ of the system, which can be calculated by subtracting the vibrational entropy, $S_{v i b}$, from the total entropy of the system,

$$
S_{c}=S_{t o t}-S_{v i b}=S_{i d e a l}+S_{e x}-S_{v i b} .
$$

The vibrational entropy is calculated by making a harmonic approximation about a local minimum. ${ }^{37,40-42}$ To obtain the vibrational frequencies, we calculate the Hessian and then diagonalize it. Once we obtain the vibrational frequencies, $S_{v i b}$ is calculated using the following equation:

$$
S_{v i b}=\frac{3}{2} \ln \left(\frac{2 \pi T}{h^{2}}\right)+\frac{\ln (V)}{N}+\frac{1}{2 N} \sum_{i=1}^{3 N-3} \ln \left(\frac{2 \pi T}{\omega_{i}^{2}}\right)-\frac{3}{2 N}+3 .
$$

\section{RESULTS FOR MEAN-FIELD SYSTEM}

In this section, we will discuss the entropy of the mean-field system and its correlation with the dynamics. We will first discuss the results obtained using the TI method and its shortcomings and then discuss the results obtained from the 2PT method. 


\section{A. Entropy using thermodynamic integration method}

In the estimation of the entropy using the TI method, we need to calculate the excess entropy and the vibrational entropy. The configurational entropy is then obtained from Eq. (28).

\section{Excess entropy}

Note that in the calculation of the excess entropy via the TI method, we need the information of the internal energy [Eq. (13)]. For the mean-field systems, the internal energy has two parts, one is the contribution from the regular nearest neighbor $(\mathrm{NN})$ and the other is the contribution from the pseudo-neighbor (PN). A similar decomposition is present for the entropy, where we can write $S_{e x}=S_{e x}^{N N}+S_{e x}^{P N}$. The first term on the rhs refers to the contribution from the regular neighbors and the second term refers to that from the pseudoneighbors. These are given by

$$
S_{e x}^{N N}\left(\beta^{*}, k\right)=\beta^{*}\langle U\rangle-\int_{0}^{\beta^{*}} d \beta\langle U\rangle
$$

and

$$
S_{e x}^{P N}\left(\beta^{*}, k\right)=\beta^{*}\left\langle U_{k}^{p s e u d o}\right\rangle-\int_{0}^{\beta^{*}} d \beta\left\langle U_{k}^{p s e u d o}\right\rangle .
$$

In Fig. 1, we plot the temperature dependence of $S_{e x}$ from the TI method for different $k$ systems. In the TI method, we assume the particles to be indistinguishable. We find that the excess entropy decreases with increasing $k$. Our earlier study showed that with increasing $k$, the structure of the system remains unchanged. ${ }^{2}$ Thus, the contribution of the regular neighbors to the entropy does not change with $k$. However, with an increase in the number of pseudoneighbors and thus $U_{k}^{p s e u d o}$, the total excess entropy decreases. Thus, the decrease in excess entropy obtained via the TI method can be attributed to the increase in the pseudoneighbor interactions.

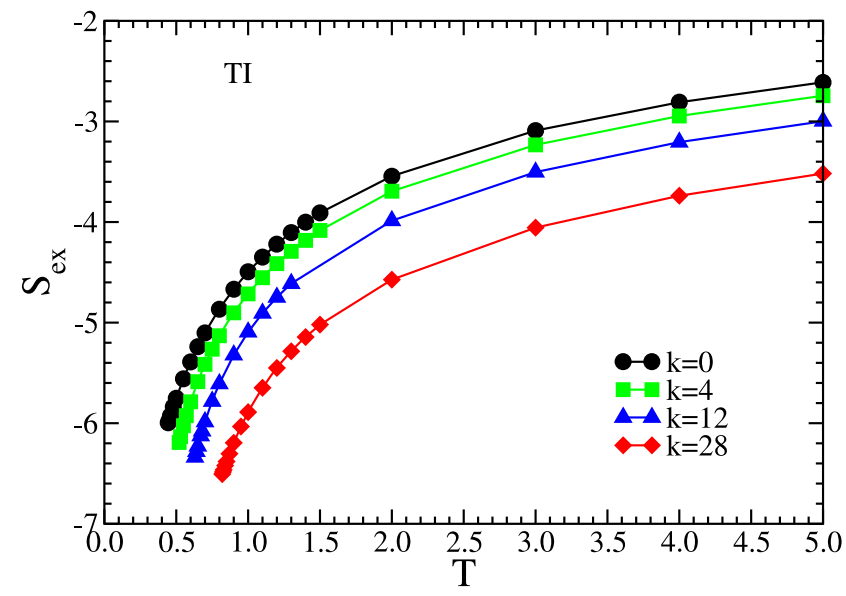

FIG. 1. Plot of per particle excess entropy $S_{\text {ex }}$ vs $T$ for $k=0,4,12$, and 28 systems. $S_{e x}$ is estimated using the TI method. With an increase in $k$, the excess entropy becomes more negative.

\section{Vibrational entropy}

We next calculate the vibrational density of states (VDOS) for different $k$ values from the Hessian. This calculation is cross-checked by calculating the VDOS independently from the Fourier transform of the velocity auto-correlation function, which matches the data obtained from the Hessian (see Appendix A).

We find that with an increase in pseudoneighbors, there is a suppression of the low-frequency modes, and the whole spectrum moves to a higher frequency range, as shown in Fig. 2. A similar effect was also seen in the high-temperature dynamics where it was shown that with the increase in the pseudoneighbors, the cage becomes stiffer and the dynamics inside the cage becomes faster.

The temperature dependence of the vibrational entropy $S_{v i b}$ (obtained from the VDOS) is plotted in Fig. 3. We find that with increasing $k$, as the vibrational spectrum shifts to higher frequencies, the vibrational entropy decreases.

For a few representative systems $(k=0$ and $k=28)$, we also show the vibrational entropy as obtained from the anharmonic approach $^{43}$ and the Frenkel and Ladd (FL) method ${ }^{44}$ [Figs. 3(b) and $3(\mathrm{c})$ ]. For comparison, we also plot the vibrational entropy as obtained from the harmonic approximation. The details of these methods are given in Appendix A. As expected, the vibrational entropy obtained from these two approaches is slightly lower than that obtained from the harmonic approach.

\section{Configurational entropy}

Next, we study the configurational entropy of the system. For all the systems, the data are plotted below their respective onset temperatures (see Table I). ${ }^{27}$ The systems follow the expected linear relationship between $T S_{c}$ and $T$ (Fig. 4). The Kauzmann temperature $T_{K}^{T I}$ is obtained by fitting to $T S_{c}=K_{T}\left(\frac{T}{T_{K}}-1\right)$. We find that $T_{K}^{T I}$ increases with $k$. This is expected as in the earlier study, it was found that with an increase in pseudoneighbors, the $\alpha$ relaxation time of the system appears to diverge at a higher temperature. ${ }^{27}$ However, for larger $k$ systems $(\mathrm{k}=12$ and 28$)$, we find negative values of the

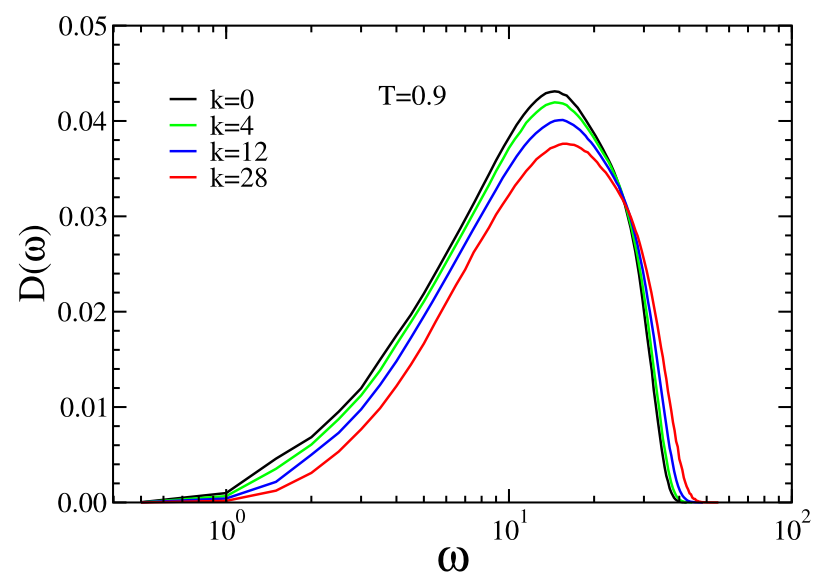

FIG. 2. Vibrational density of states (VDOS), $D(\omega)$ vs $\omega$, for $k=0,4,12$, and 28 systems. With the increase in $k$, the low-frequency modes are suppressed and the whole spectrum shifts to higher frequencies. 

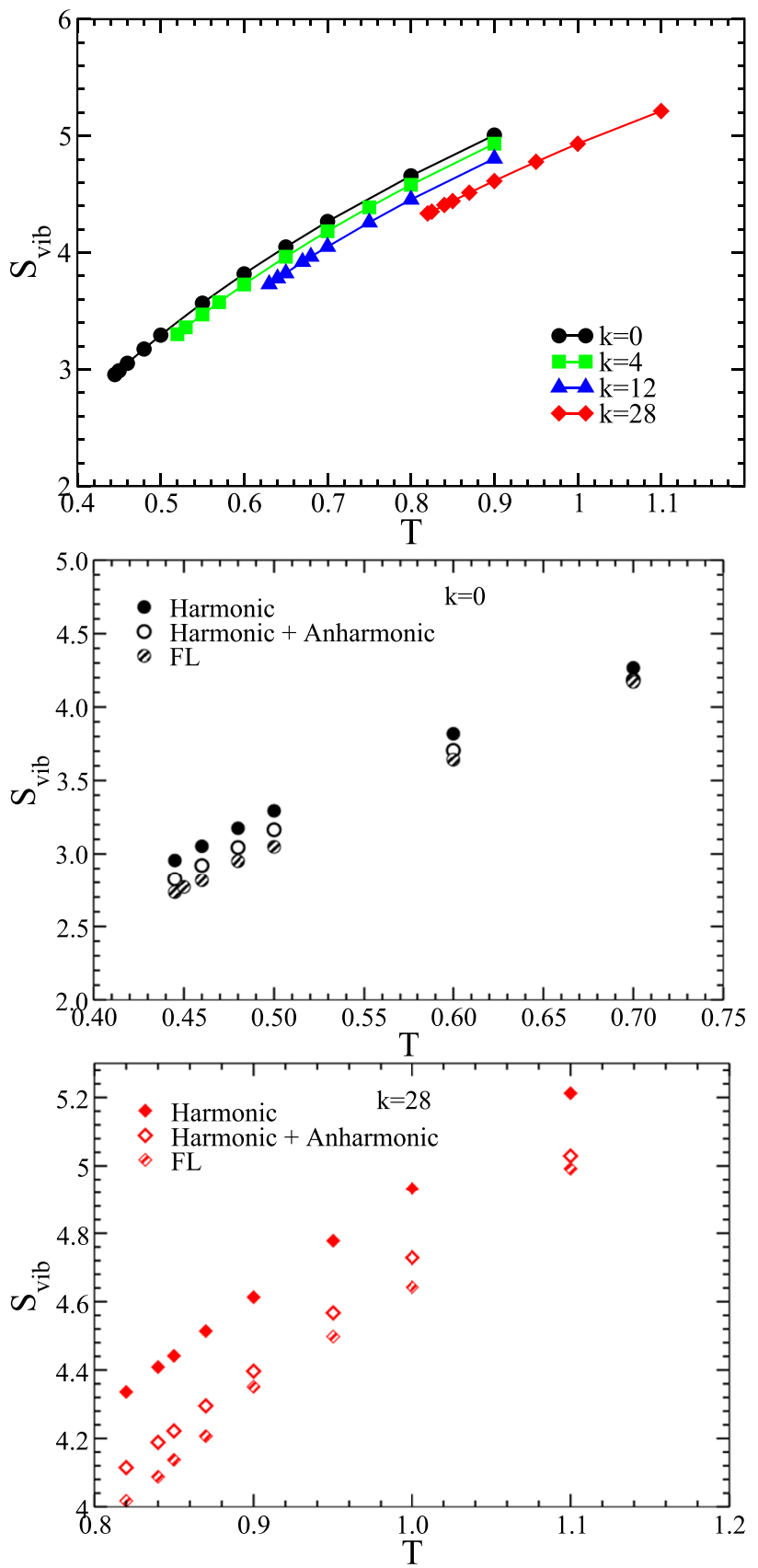

FIG. 3. (a) The vibrational entropy $S_{v i b}$ vs $T$ for $k=0,4,12$, and 28 systems. With an increase in $k$, the VDOS shifts to higher frequencies, leading to a decrease in the vibrational entropy. The vibrational entropy using the Frenkel-Ladd method and using the anharmonic contribution for (b) $k=0$ and (c) $k=28$ systems. The estimates by the two methods are closer to each other compared to the harmonic approximation.

configurational entropy at comparatively high temperatures where the liquid can be equilibrated in simulations. Particularly for the $k=28$ system, the temperature where the configurational entropy vanishes is close to the onset temperature of glassy dynamics. ${ }^{27}$ This
TABLE I. Values of all characteristic temperatures for systems with different $k$ values. $T_{0}$ is the VFT temperature where the $\alpha$ relaxation time diverges according to fits to the VFT equation, Eq. (10). $T_{K}^{T I}$ is the Kauzmann temperature estimated from TI. $T_{K}^{2 P T}$ is the Kauzmann temperature estimated from the 2PT method.

\begin{tabular}{lcccc}
\hline \hline $\mathrm{k}$ & $\mathrm{T}_{\text {onset }}$ & $\mathrm{T}_{0}$ & $\mathrm{~T}_{K}^{T I}$ & $\mathrm{~T}_{K}^{2 P T}$ \\
\hline 0 & $0.74 \pm 0.04$ & 0.28 & 0.28 & 0.24 \\
4 & $0.83 \pm 0.08$ & 0.36 & 0.46 & 0.31 \\
12 & $1.03 \pm 0.07$ & 0.46 & 0.68 & 0.41 \\
28 & $1.28 \pm 0.22$ & 0.61 & 1.19 & 0.55 \\
\hline \hline
\end{tabular}

is clearly unphysical. The $T_{K}^{T I}$ values are listed in Table I. In Table I, we also list the respective $T_{0}$ values. For many systems, it is found that $T_{K} \simeq T_{0}$, which suggests that the slowing down of the dynamics is driven by thermodynamics. ${ }^{14}$ On the contrary, in Table I, we find
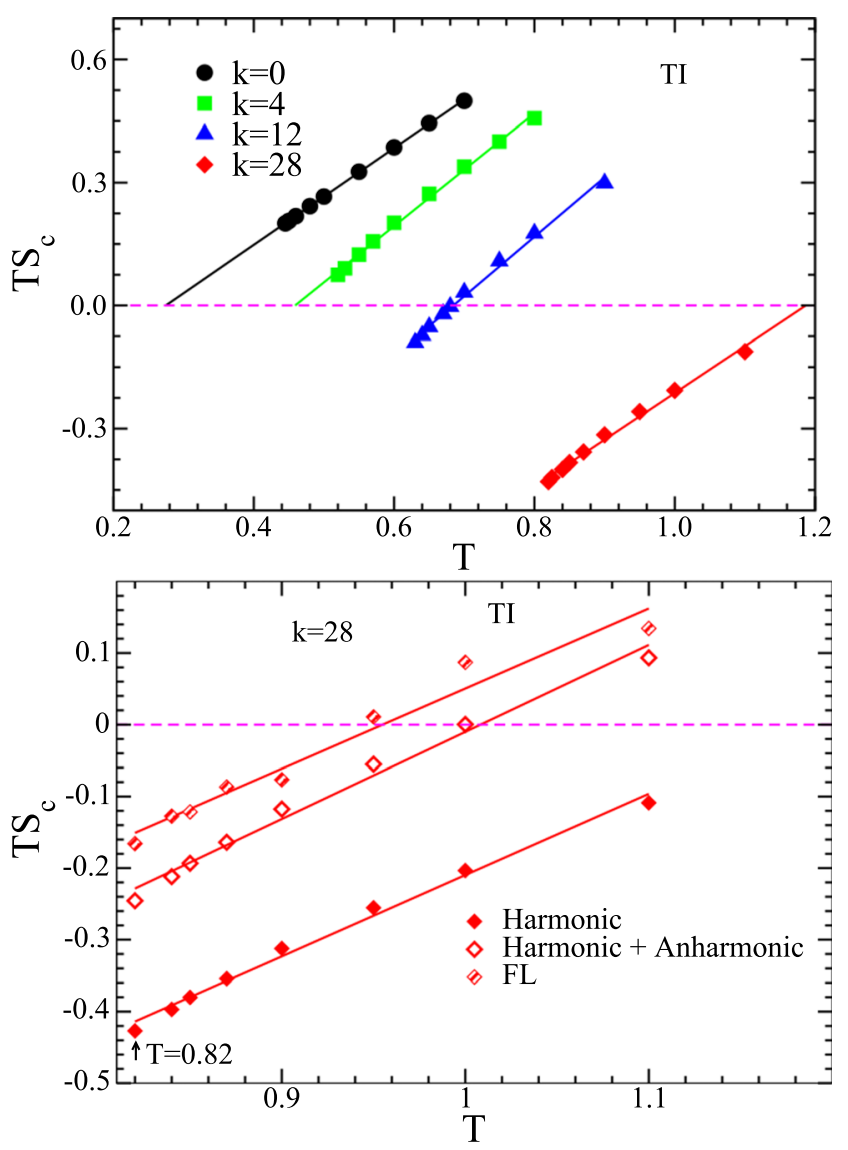

FIG. 4. (a) $T S_{c}$ vs $T$ for $k=0,4,12$, and 28 systems where $S_{c}$ is calculated using the TI method. The value of the Kauzmann temperature $T_{K}^{T /}$ increases with increasing $k$. The value of $T_{K}^{T \prime}$ (see Table I) for the $k=28$ system is close to its onset temperature. For $k=12,28$ systems, $T_{K}^{T /}$ values are high enough such that temperatures below $T_{K}^{T I}$ are accessible in simulation. $S_{c}$ becomes negative for such temperatures. (b) $T S_{c}$ vs $T$ for the $k=28$ system. Here, three different vibrational entropies have been estimated using the pure harmonic approximation, including the anharmonic contribution to the harmonic approximation and the Frenkel-Ladd method. All three methods predict negative $S_{c}$ below a certain temperature, where the dynamics of the system survives. 
that the difference between $T_{K}^{T I}$ and $T_{0}$ increases with an increase in $k$ and the former is higher than the latter.

In the TI method, we need information about the ideal gas entropy, the excess entropy, and the vibrational entropy. To improve the estimate of the vibrational entropy, we also incorporate the anharmonic contribution and calculate the vibrational entropy using the Frenkel and Ladd method (Appendix A). In Fig. 4, we plot $T S_{c}$ for the $\mathrm{k}=28$ system where the vibrational entropy is obtained from the anharmonic approximation and the Frenkel and Ladd method. For comparison, we plot the value as obtained from the harmonic approximation. We find that although the configurational entropy now vanishes at a lower temperature, its value is still negative at temperatures where we can equilibrate the system.

We next show in Fig. 5 that for the $k=28$ system at $T=0.82$, which is much below $T_{K}^{T I}=1.19$, both the collective overlap function and the intermediate scattering function decay with time and reach their respective long time values $\left[Q^{\text {tot }}(t \rightarrow \infty)=0.135\right.$ and $F(q, t \rightarrow \infty)=0]$. Note that because of the introduction of the pseudoneighbors at a distance " $L_{i j}$," the system has more than one length scale. Thus, to make sure that the relaxation persists at length scales that are larger and smaller than the nearest neighbor distance, we plot the intermediate scattering function at wave numbers larger and smaller than $q_{\max }=\frac{2 \pi}{\sigma_{\max }}$, where $\sigma_{\max }$ is the position of the first peak in the radial distribution function. We find that the intermediate scattering functions relax to zero at all length scales. The fact that the dynamics show full relaxation where the configurational entropy vanishes suggests a strong violation of the RFOT prediction. Note that recent studies have suggested that the TI method overestimates the configurational entropy. ${ }^{45,46}$ If we take this into account, i.e., assume that the actual configurational entropy is lower than that obtained via the TI method, then the results obtained here

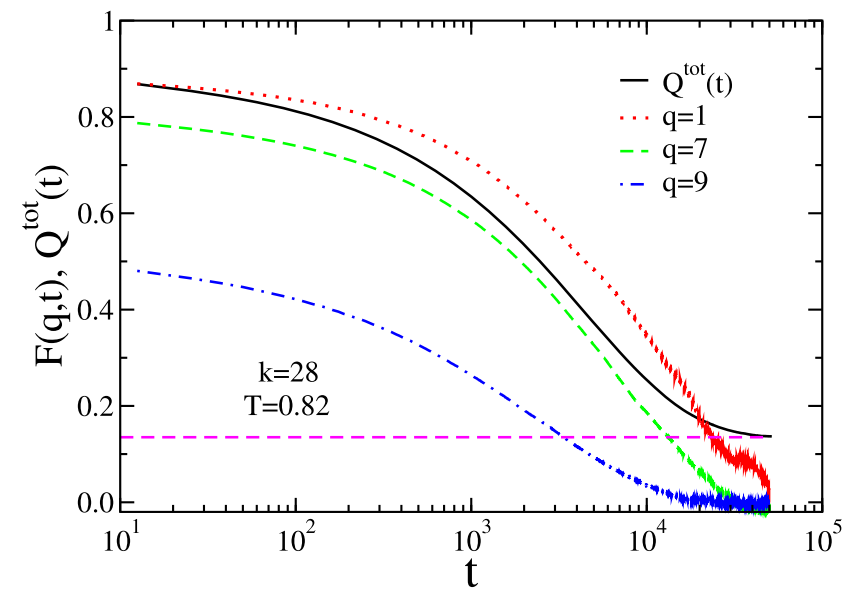

FIG. 5. Time dependence of the intermediate scattering function and the collective overlap function for the $k=28$ system at a temperature $T=0.82$, which is lower than $T_{K}^{T I}$ (see Table I). It shows that the self-dynamics and the collective dynamics relax to their asymptotic values over timescales accessible in simulations at a temperature lower than that at which the configurational entropy vanishes. The magenta horizontal dashed line shows the asymptotic value of the collective overlap function, which is 0.135 .

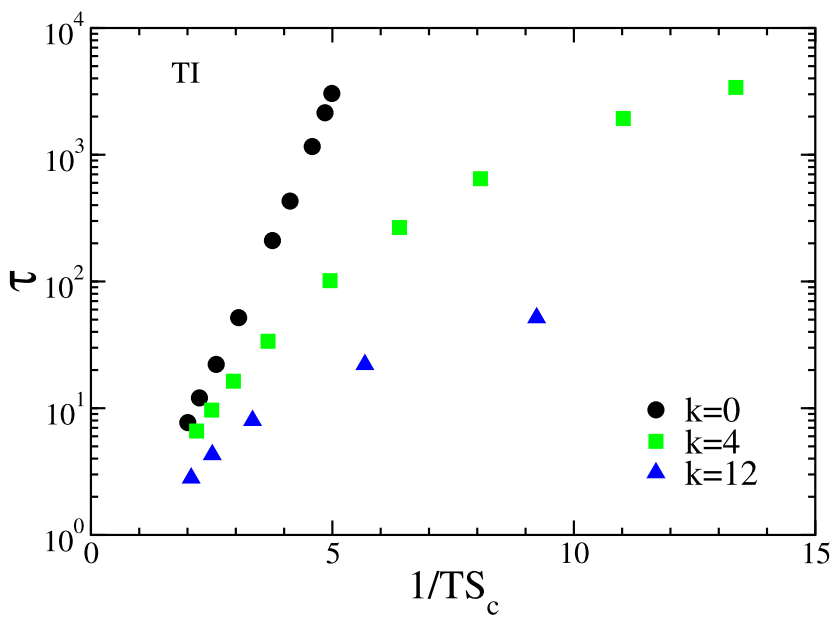

FIG. 6. Testing the Adam-Gibbs relation between the relaxation time $\tau$ and $1 / T S_{c}$, for the $k=0,4$, and 12 systems. The $A G$ relation is obeyed for the $k=0$ system, but is violated for non-zero $k$ systems. The relaxation time $\tau$ is estimated from the self-part of the overlap function.

would be even more flawed. This implies that we need to revisit the TI method of calculating the entropy.

In Fig. 6, we also study the validity of the Adam-Gibbs relationship. We find that with an increase in $k$, there is a breakdown of the AG relationship, which in this case is a consequence of the violation of RFOT predictions. The plot also suggests that the relaxation time is smaller than that predicted by the AG relationship.

\section{B. Possible reasons for the failure of the TI method}

Let us first summarize the main observations made here when the entropy is calculated using the TI method: (i) negative values of $S_{c}$ at low temperatures for large values of $k$ and (ii) full relaxation of the dynamical quantities at temperatures lower than the temperature at which $S_{c}$ goes to zero. In this section, we discuss the possible failure points of the TI method.

\section{Ideal gas entropy}

In the calculation of the configurational entropy [Eq. (28)], we need the information of the ideal gas entropy. To make the entropy an extensive quantity, we calculate the ideal gas entropy (Sec. III A 1) by assuming the particles to be indistinguishable. However, in the mean-field system, each particle has a different set of pseudoneighbors with different $L$ values. Thus, one might argue that the particles are distinguishable.

If we assume all particles to be distinguishable, i.e., $M=N$, then the entropy in the thermodynamic limit will diverge [Eq. (12)]. However, for finite $N$, we can estimate the entropy, which will increase by a factor that is proportional to $\log (N)$ but independent of $k$. From our analysis, it appears that with an increase in $k$, the error in the entropy calculation increases. This implies that the correction term should depend on $k$.

Apart from the distinguishability factor, there is one other issue that can affect the ideal gas term. Here, the way the interaction 
between a particle and its pseudoneighbor is designed restricts the particle to access a certain part of the total volume. Per pseudoneighbor this volume is a spherical region of radius $L_{i j}$. Thus, in the ideal gas limit, the whole volume of the system is not accessible to a particle. The per particle inaccessible volume should increase with $k$, which will lower the entropy of the system. Thus, the distinguishability factor will increase the entropy, whereas inaccessible volume will decrease the entropy, the former is independent of $k$, but the latter depends on $k$. This might appear to solve the $k$ dependence of the correction term. However, if we combine the distinguishability and inaccessible volume part, then we will find that for systems with small values of $k$, the volume correction is really small and the distinguishability factor which is independent of $k$ increases the entropy by a large amount. Thus, the dynamics for these systems will be similar to the $k=0$ system, but the entropy calculated in this way will be much higher.

Another possibility is that the distinguishability is not a binary function but is a function of $k$. Having these extra connections with the pseudoneighbors replacing particles with another one while keeping the identity of pseudocontacts the same can increase the energy of the system, and the larger the number of pseudocontacts the higher is the increase in the energy. This appears quite similar to the case of polydisperse systems with continuous polydispersity where depending on the size range of the two particles, the replacement may or may not keep the system in the same minimum. ${ }^{47}$ It was argued that after particle swapping if the system remains in the same inherent structure minimum, then the two particles are indistinguishable, and if not, then they belong to different species. Thus, to find the number of species, we need to swap particle positions. Swapping particles while keeping the identity of the pseudoneighbors the same is not straightforward. The swap should make sure that in the new position of the particle, none of the pseudoneighbors are within the interaction range $r_{c}$. With the increase in the number of pseudoneighbors, these swaps will be mostly rejected, thus making it impossible to quantify the number of species and, thus, the entropy.

\section{Excess entropy scaling}

We next test the accuracy of the excess entropy value calculated via the TI method. Apart from the AG relationship which is valid in the low temperature regime and connects the configurational entropy to the dynamics, there is another phenomenological relationship, namely, the Rosenfeld relation between the excess entropy and the dynamics. ${ }^{48,49}$ According to the Rosenfeld relation, any dimensionless transport property will follow the excess entropy scaling. For the relaxation time, it can be written as $\tau^{*}=R \exp \left(-K S_{e x}\right)$, where $\tau^{*}=\tau \rho^{-1 / 3} T^{1 / 2} m^{-1 / 2}$. For simple liquids, it has been found that $R \simeq 0.6$ and $K \simeq 0.8$, and this relationship is valid in the high-temperature regime, showing a data collapse between scaled diffusion and $S_{e x}{ }^{50}$ and also scaled relaxation time and $S_{\text {ex }}{ }^{51,52}$ A recent study has also shown that scaled viscosity and diffusion coefficient for a large number of systems show a quasiuniversal excess entropy scaling, extending over both high and low temperature regimes. ${ }^{53}$ In Fig. 7 , we plot $\tau^{*}$ vs $S_{\text {ex }}$ for the different mean-field systems and do not find any data collapse. Thus, we find a breakdown of the Rosenfeld relation and also of the quasi universal excess entropy scaling. ${ }^{53}$ Note that similar to that observed for the

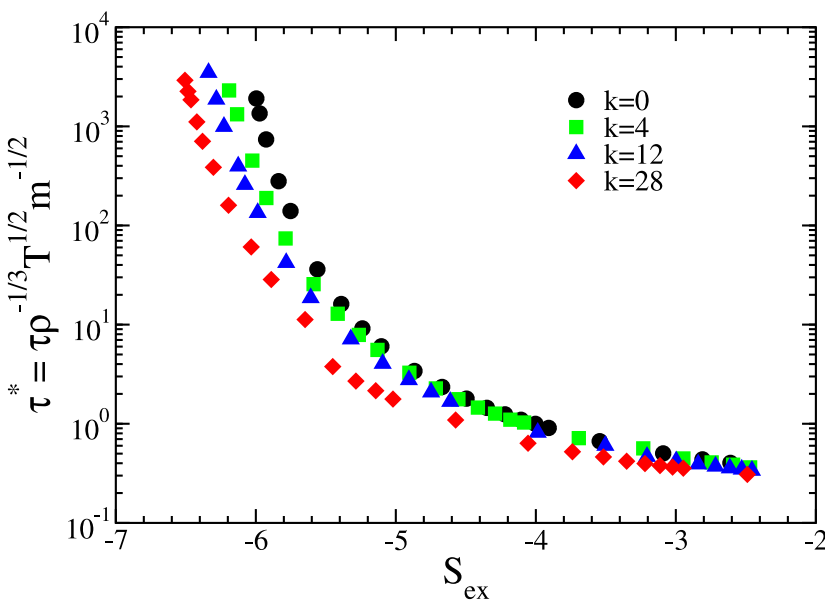

FIG. 7. Scaled relaxation time vs excess entropy. With increasing $k$, there is a deviation from the quasi-universal excess entropy scaling.

AG relationship, we find that for higher $k$ systems, the relaxation time is smaller than that predicted by the excess entropy scaling relation.

The deviation from the excess entropy scaling might appear quite weak. However, note that, unlike the AG relationship where we deal with the configurational entropy which has a very small value, here, we deal with the excess entropy, which has a large value. Thus, the excess entropy scaling is less sensitive to small errors in the calculation of the entropy. In the mean-field system, we find that the excess entropy has a strong dependence on the number of pseudoneighbors. On the other hand, the study of the dynamics of the mean-field system showed that the interaction with the pseudoneighbors slows down the overall dynamics of the system but has a weak effect on the structural relaxation. ${ }^{27}$ Thus, it appears that the role of the pseudoneighbors is not the same for the TI entropy and the dynamics.

\section{Entropy using the 2PT method}

Although we point out the possible sources of error in the TI method, we do not know how to correct them at present. Thus, in this section, we present the results of the calculation of entropy using the $2 \mathrm{PT}$ method, which uses an entirely different technique. In the 2PT method, we primarily use information about the dynamics, namely, the velocity autocorrelation function, to determine the entropy. We know that the TI method works well for the regular KA model. Thus, to validate the $2 \mathrm{PT}$ method, we compare it with the TI method for a regular KA system $(k=0)$. As shown in Appendix A, the $2 \mathrm{PT}$ method works well. At temperatures close to the mode-coupling transition temperature, the $2 \mathrm{PT}$ method shows some deviation, which is identified as arising from an averaging issue. Thus, we use the results from the 2PT method in the temperature range where the upper bound is the onset temperature and the lower bound is above the respective mode-coupling theory transition temperature. ${ }^{27}$ In this section, we will first compare the total entropy obtained using the 2PT method [Eq. (27)] and the TI [Eq. (14)] method for the different mean-field systems. As shown in 

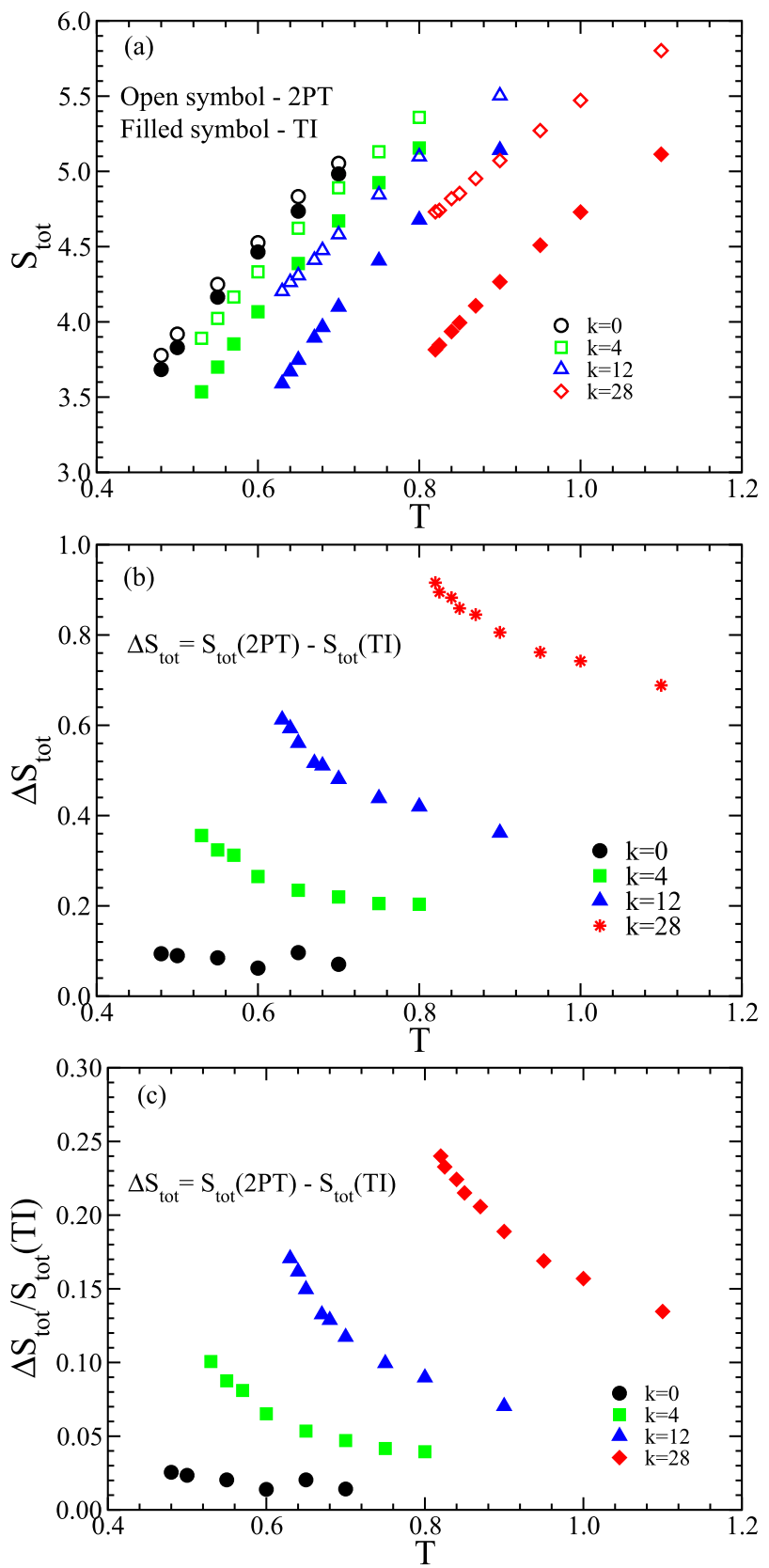

FIG. 8. Comparison of the TI and 2PT methods of calculation of the entropy for the mean-field system: (a) $S_{\text {tot }}$ vs $T$. The filled symbols represent the results obtained from the TI method, and the open symbols represent those from the 2PT method. $S_{\text {tot }}$ computed by the 2PT method is higher than that by the TI method. (b) The difference in total entropy, $\Delta S_{\text {tot }}$, between 2PT and TI methods increases with increasing $k$. (c) The relative difference in the total entropy, $\frac{\Delta S_{\text {tot }}}{S_{\text {tot }}(T /)}$, between 2PT and $\mathrm{TI}$ methods shows similar behavior as (b).

Fig. 8, the difference in total entropy between TI and 2PT methods increases systematically with increasing $k$. This suggests that for this system, the TI method of calculating the entropy is not correct. We next study the configurational entropy as predicted by the $2 \mathrm{PT}$

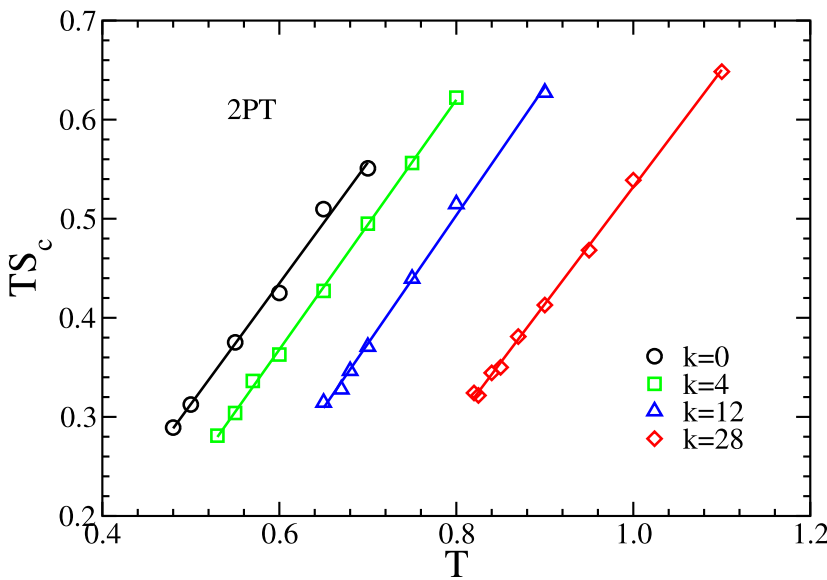

FIG. 9. $T S_{c}$ vs $T$ for $k=0,4,12$, and 28 systems using the 2PT method. Values of $T_{K}^{2 P T}$, which are close to $T_{0}$, are given in Table I.

method and its correlation with the dynamics. To calculate the configurational entropy, we need the information of the vibrational entropy, which is the same as that used in the TI method. In Fig. 9, we show the $T S_{c}$ vs $\mathrm{T}$ plots. We find that for all the systems, $T_{K}^{2 P T}$ is smaller than $T_{K}^{T I}$ and close to $T_{0}$ (see Table I). This shows that the entropy calculated via the $2 \mathrm{PT}$ method follows the RFOT prediction. As discussed before, recent studies suggested that the TI method overestimates the entropy. ${ }^{45,46}$ This might imply that the higher value of entropy estimated by the $2 \mathrm{PT}$ method is due to some additional vibrational degrees of freedom. Note that, for the $k=0$ system, the entropy estimated by the two methods is close to each other. However, with the increase in $k$, as the error in the entropy value calculated by the TI method increases, this difference also increases. For the $k=28$ system, when the TI method predicts

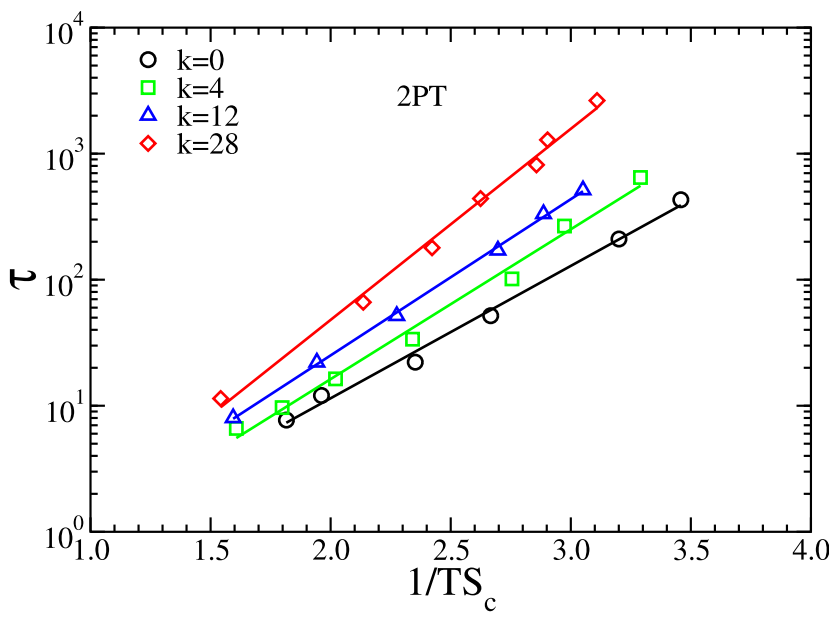

FIG. 10. Testing the AG relation, $\tau$ vs $\frac{1}{T S_{c}}$, for $k=0,4,12$, and 28 systems with $S_{c}$ computed by the $2 \mathrm{PT}$ method. All the systems follow the AG relation in the range of temperatures studied here. 
negative configurational entropy and the $2 \mathrm{PT}$ method predicts positive configurational entropy, the self-correlation and the collective correlation functions decay to their respective asymptotic values. This decay of the correlation functions is only possible when the system can explore the configurational space. Vibrational degrees of freedom alone cannot lead to such a decay of the correlation functions. This is consistent with the prediction of the $2 \mathrm{PT}$ method.

We next check the validity of the AG relationship. In Fig. 10, we show a semi-log plot of $\tau$ against $\frac{1}{T S_{i}}$. It clearly shows the validity of the AG relation for all the systems in the temperature range studied. Note that in the $2 \mathrm{PT}$ method, there is no adjustable parameter to enforce the validity of the AG relationship.

\section{RESULTS FOR PINNED SYSTEMS}

Note that in the mean-field system, the breakdown of the AG relation and also the vanishing of the configurational entropy at a temperature where the dynamics show complete relaxation are similar to what has been observed for another family of models, namely, the pinned system. ${ }^{33-36,45}$ In the pinned system, the relaxation time obtained from single-particle dynamics remains finite at temperatures for which the configurational entropy vanishes, and there is some evidence ${ }^{54}$ that the relaxation time associated with the collective dynamics also remains finite at such temperatures. It has also been argued that the configurational entropy has a finite value when the vibrational entropy is calculated using an anharmonic approximation. ${ }^{4}$

We calculate the total entropy of the pinned system using the TI method used in earlier studies ${ }^{33}$ and also given in Appendix B of the present paper. We then calculate the configurational entropy by subtracting the vibration entropy from the total entropy by taking into consideration the anharmonic contribution. As discussed in Appendix B and shown in Figs. 21 and 22 and Table II, even after taking into consideration the anharmonic term, the Kauzmann temperature $T_{K}$ appears to be high and the AG relationship is violated.

Given the success of the 2PT method in determining the entropy for the mean-field system, we apply it for the pinned system and compare it with the TI method. In Fig. 11(a), we plot the total entropy obtained using two different methods, and in Fig. 11(b), we plot their differences for three different pinning densities, and in Fig. 11(c), we plot the relative difference. For comparison, we also show the KA system with no pinning, which is the same as the KA system with $k=0$. Similar to that observed in the mean-field system, we find a difference between the entropy calculated via the

TABLE II. The values of all characteristic temperatures for pinned systems with different pin concentration c. $T_{K}^{T I}$ is the Kauzmann temperature estimated from TI. $T_{K}^{2 P T}$ is the Kauzmann temperature estimated from the 2PT method.

\begin{tabular}{lcc}
\hline \hline $\mathrm{c}$ & $\mathrm{T}_{K}^{T I}$ & $\mathrm{~T}_{K}^{2 P T}$ \\
\hline 0.00 & 0.28 & 0.24 \\
0.05 & 0.31 & 0.30 \\
0.10 & 0.41 & 0.32 \\
0.15 & 0.57 & 0.41 \\
\hline \hline
\end{tabular}
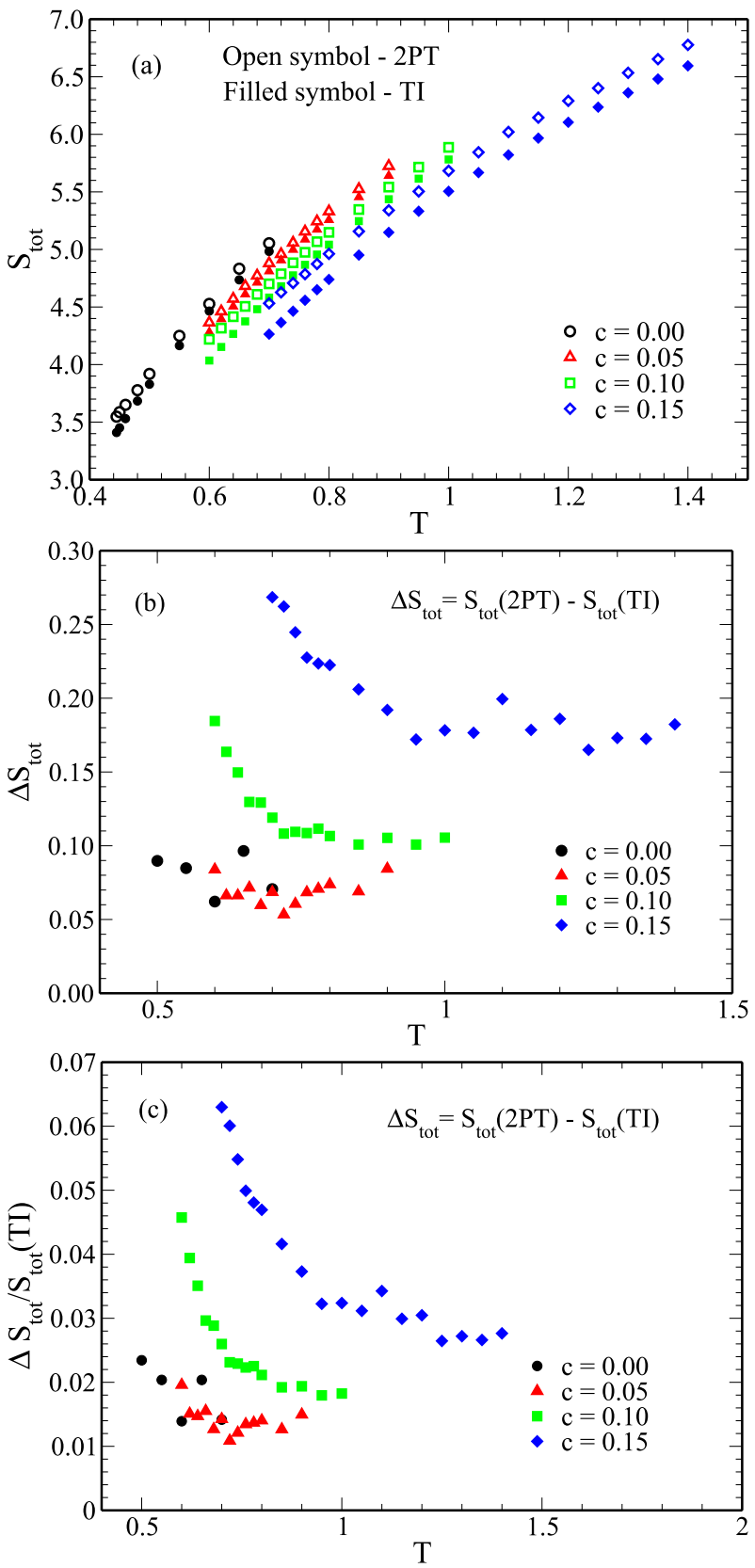

FIG. 11. Comparison of the TI and 2PT methods of calculation of entropy for the pinned system with different pinning concentration. (a) The total entropy $S_{\text {tot }}$ VsT . The filled symbols represent the results of the TI method, and the open symbols represent those of the 2PT method. (b) The difference in $S_{\text {tot }}$ between 2PT and TI methods increases with increasing pinning concentration $c$. (c) The relative difference in the total entropy, $\frac{\Delta S_{\text {tot }}}{S_{\text {tot }}(T I)}$, between 2PT and TI shows similar behavior as (b)

2PT and the TI methods that increases systematically with pinning. We next calculate the configurational entropy as predicted by the two methods and plot the temperature dependence of $T S_{c}$ in Fig. 12. Both methods predict positive Kauzamnn temperatures for 

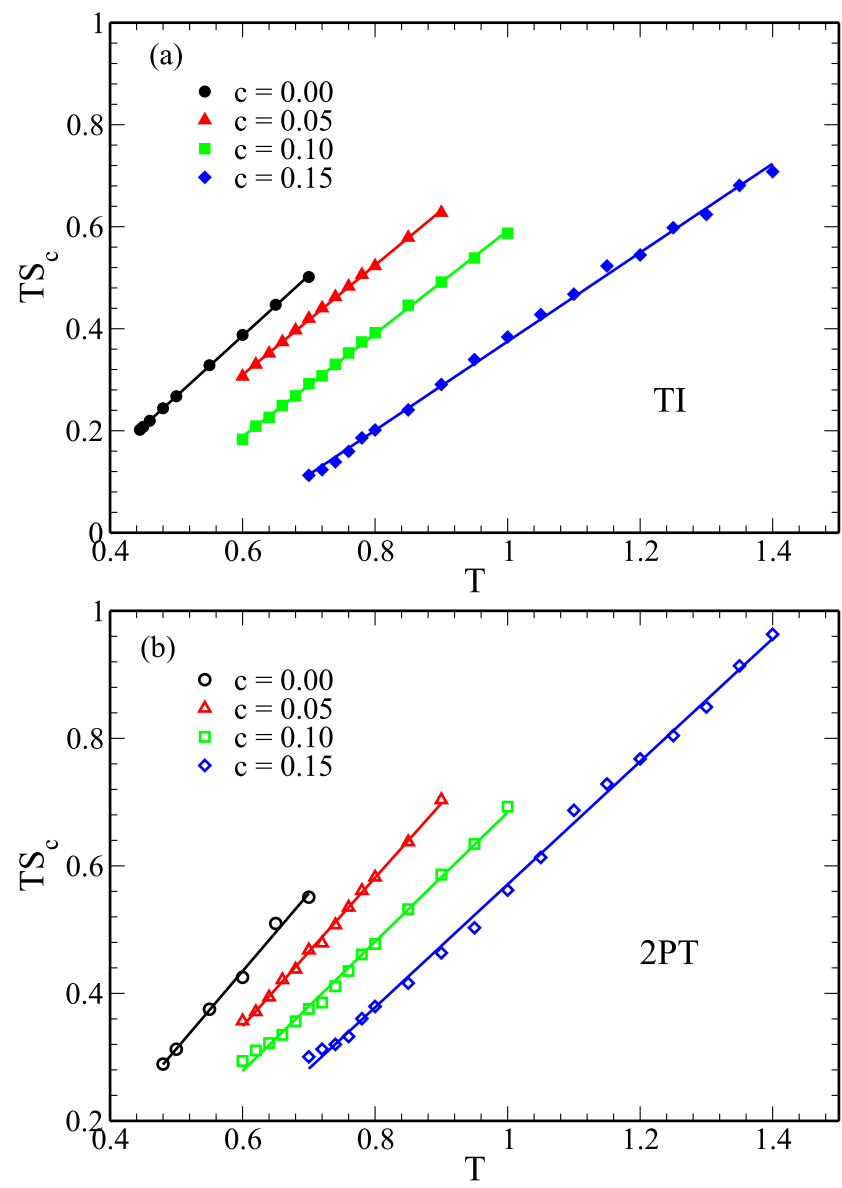

FIG. 12. $T S_{c}$ vs $T$ for systems with different pinning concentrations $c=0,0.5$ $0.10,0.15$ using (a) the TI method and (b) the 2PT method. Both $T_{K}^{T I}$ and $T_{K}^{2 P T}$ increase with increasing pinning concentration, but $T_{K}^{2 P T}<T_{K}^{T /}$; see Table II.

each system, and similar to the case of mean-field systems, the Kauzmann temperature predicted by the $2 \mathrm{PT}$ method is lower than that by the TI method; see Table II. In this calculation, we have used the harmonic approximation for the vibrational entropy. The anharmonic approximation will equally affect both the $2 \mathrm{PT}$ and TI entropy values, and the plots are given in Appendix B.

Next, we need to understand if the lowering of the $T_{K}$ value in the $2 \mathrm{PT}$ method is sufficient to describe the dynamics. In Fig. 13, we show semi-log plots of $\tau$ vs $\frac{1}{T S_{c}}$ where the entropy is calculated using the $2 \mathrm{PT}$ and the TI methods. The TI method shows a strong breakdown of the AG relation for $c=0.1$ and $c=0.15$, whereas the 2PT method clearly follows the AG relation for all $c$.

As discussed above, the unphysical feature of negative configurational entropy does not appear in the TI results for the pinned system. In addition, the observation that the $2 \mathrm{PT}$ results for $S_{c}$ lie above the results obtained from the TI method suggests that the $2 \mathrm{PT}$ results are less accurate than the TI results because, as mentioned above, there are arguments ${ }^{45,46}$ that suggest that actual configurational entropy is lower than the TI result. However, it should be noted that the expectation that the TI results overestimate
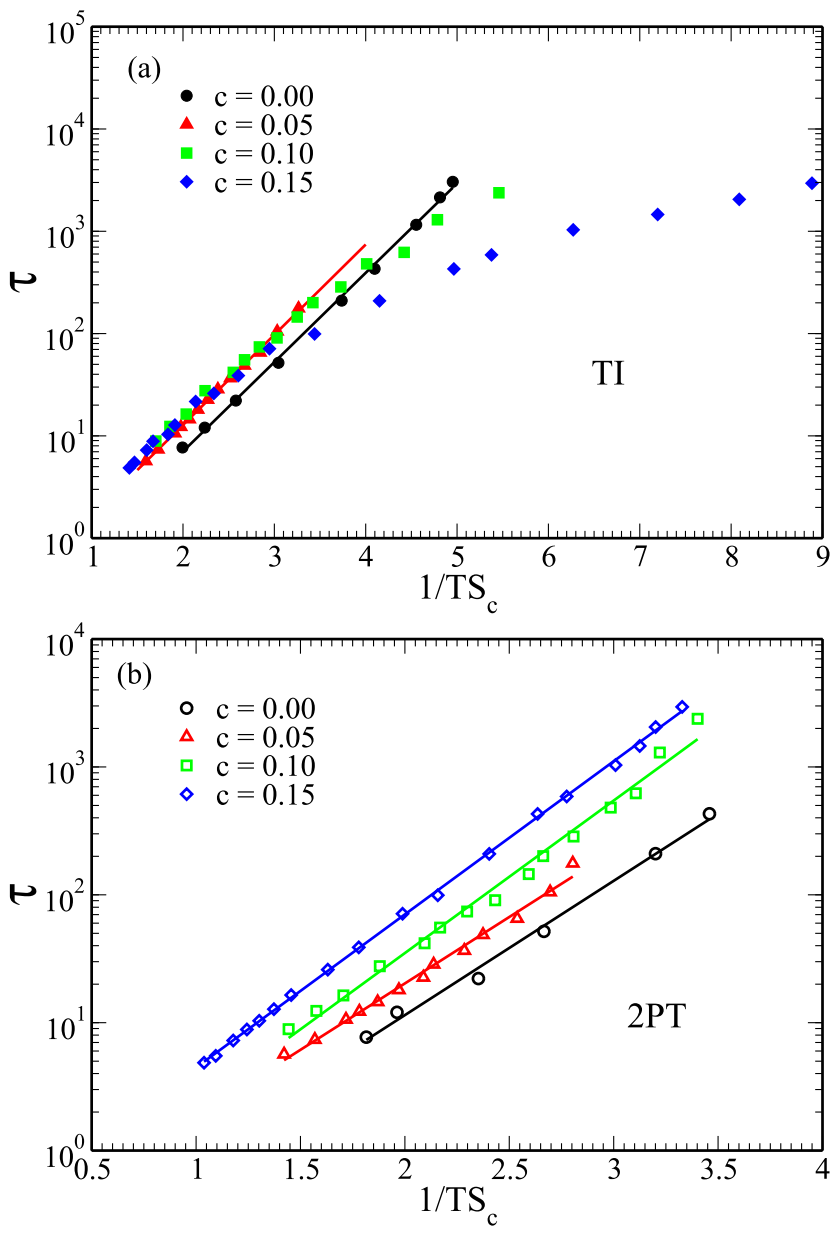

FIG. 13. Testing the AG relation between $\tau$ vs $\frac{1}{T S_{c}}$ for $c=0,0.5,0.10,0.15$ systems using (a) the TI method (b) the 2PT method. In the temperature range studied here, the $A G$ relation is violated for $c=0.1$ and $c=0.15$ when $S_{c}$ is calculated using the TI method. However, the AG relation holds for all $c$ when $S_{c}$ is calculated via the 2PT method.

the true $S_{c}$ has not been verified by any calculation for the pinned system. In addition, a difference between the value of the configurational entropy per particle obtained from the TI method and its actual value would require the presence of an exponentially large number of energy minima that differ from a particular minimum by the movement of a small number of particles. We are not aware of any study that establishes the presence of such a large number (exponential in the number of particles) of nearby minima.

Available results for the dynamics ${ }^{35,36,54}$ of the pinned system suggest that the TI results for $S_{c}$ may not be consistent with the RFOT theory. There are arguments ${ }^{34,45}$ that suggest that the inconsistency with the RFOT theory may disappear if the relaxation time associated with the collective dynamics (instead of the timescale obtained from single-particle dynamics) is considered. It has also been argued $^{45}$ that the disagreement with the RFOT theory may be accounted for by the overestimation of $S_{c}$ in the 
TI method. However, these arguments have not been verified from explicit calculations.

A similar observation of the mismatch between entropy and dynamics for the pinned system has been observed in experimental studies. ${ }^{55}$ It was found that although for unpinned systems, the local dynamics correlate with the local pair excess entropy, and with an increase in the pinning density, such correlation disappears. ${ }^{55}$ Thus, for the pinned system, these traditional methods of entropy calculation cannot explain the dynamics. On the other hand, the 2PT method appears to explain the dynamics quite well. For these reasons, we believe that more studies are necessary for deciding the relative merits of the TI and 2PT methods for the pinned system.

\section{CONCLUSION}

Recently, some of us have developed a model for a glassforming liquid whereby changing a parameter, the system can continuously switch from a standard three-dimensional liquid to a fully connected mean-field-like system. ${ }^{27}$ The parameter is $k$, the number of additional particle-particle interactions that are introduced per particle on top of the regular interactions in the system. With increasing $k$, the structure and the dynamics were studied, which showed more mean-field-like behavior at higher $k$ values. The present work aims at studying the thermodynamics of the system and understanding its correlation with the dynamics. To study thermodynamics, we first calculate the entropy using the well-known TI method. ${ }^{29}$ We then study the correlation of the entropy with the dynamics. This model shows super-Arrhenius dynamics similar to conventional glassy liquids, ${ }^{27}$ suggesting that the RFOT description should apply. However, we find that configurational entropy vanishes at a temperature that is close to the onset temperature of the system. We also find that the relaxation times calculated from both single-particle and collective dynamics remain finite at temperatures where the configurational entropy vanishes. This is different from the prediction of RFOT and the behavior seen in conventional glass-forming liquids for which the (extrapolated) values of $T_{K}$ and $T_{0}$ are found to be close to each other. ${ }^{14,16,56}$ This observation also indicates that the system apparently samples state point with negative configurational entropy, which is unphysical by definition. We discuss the possible source of error in the TI method of calculation of the entropy for the mean-field system. However, at this point, we do not know how to modify the TI method to correctly calculate the entropy of these model systems.

We, thus, use another technique, namely, the 2PT method, to calculate the entropy. The $2 \mathrm{PT}$ method assumes that a liquid can be represented as partially a gas and partially a solid, and this fraction is a function of the thermodynamic parameters of the system and also of the size of the particles. The 2PT method has been extensively used to calculate the entropy for many systems, mostly in the high-temperature regime. ${ }^{30,39}$ In recent work, this method was also extended to lower temperatures. ${ }^{32}$ We find that for the KA system at $k=0$, both the $2 \mathrm{PT}$ method and the TI method provide similar results. We then compare the total entropy calculated by the $2 \mathrm{PT}$ method with that by the TI method for different mean-field systems. We find that the difference between the entropy values obtained in the two methods systematically increases with increasing $k$. We also find that the entropy calculated via the $2 \mathrm{PT}$ method describes the dynamics quite well and confirms the RFOT prediction.
The results of the mean-field systems appear quite similar to that of the pinned particle system studied earlier. ${ }^{33}$ In the pinned system, the self-part of the density correlation function decays to zero at temperatures where $S_{c}$ obtained from the TI method goes to zero. ${ }^{35}$ Given the success of the 2PT method in calculating the entropy of the mean-field system, we apply it to calculate the entropy of the pinned system. Interestingly, we find that similar to the mean-field system, the difference between the entropy calculated via $2 \mathrm{PT}$ and TI methods systematically increases with pinning. The entropy obtained via the $2 \mathrm{PT}$ method seems to explain the temperature dependence of the relaxation time obtained from the self-overlap function well, and the RFOT prediction remains valid. However, due to reasons mentioned in Sec. V, we believe that more work is needed in order to decide whether the $2 \mathrm{PT}$ method yields more accurate results for the entropy of the pinned system than the TI method.

\section{ACKNOWLEDGMENTS}

S.M.B. thanks Walter Kob, Misaki Ozawa, Shiang-Tai Lin, Daan Frenkel, Srikanth Sastry, and Anshul D. S. Parmar for discussion and acknowledges funding support from the Science and Engineering Research Board, Department of Science and Technology, Government of India. U.K.N., P.P., and M.M. thank CSIR for the fellowship. C.D. acknowledges support from the Department of Science and Technology, Government of India.

\section{AUTHOR DECLARATIONS}

\section{Conflict of Interest}

We have no conflicts of interest to disclose.

\section{Author Contributions}

U.K.N. and P.P. equally contributed to this work.

\section{DATA AVAILABILITY}

The data that support the findings of this study are available from the corresponding author upon reasonable request.

\section{APPENDIX A: MEAN-FIELD SYSTEM ENTROPY}

For a binary system in the 2PT method of entropy calculation, we need to provide the information of the partial volume fraction, which can be calculated as ${ }^{39}$

$$
\bar{V}_{i}=\frac{\sigma_{i}^{3}}{\sum_{j} x_{j} \sigma_{j}^{3}} \frac{V}{N},
$$

where $V_{i}=\bar{V}_{i} N_{i}$

The partial volume fraction depends on the radii of the particles. In the KA system, the diameter of the $A$ and $B$ particles is 1 and 0.88 , respectively. However, the potential in the KA model is designed in such a way that it allows for interpenetration between the $A$ and the $B$ particles $\left[\sigma_{A B}<\left(\sigma_{A}+\sigma_{B}\right) / 2\right]$. Thus, if we assume that the $B$ particles are surrounded by all $A$ particles, then the effective diameter of a $B$ particle will be 0.6 . To understand the role of partial volume fraction on the entropy, we calculated $S_{\text {tot }}$ from 
the $2 \mathrm{PT}$ method, assuming the $B$ particle diameter to be 0.8 and 0.6 , respectively. We find that at high temperatures, the 0.6 value provides a better result, but at low temperatures, the entropy is almost independent of the small changes in the partial volume fraction. Thus, for these systems, we assume the diameter of the $B$ particles to be 0.6 .

We compare the total entropy of the system as estimated from the $\mathrm{TI}^{40}$ and from the $2 \mathrm{PT}^{30}$ methods. Figure 14 shows that $S_{\text {tot }}$ obtained from TI and 2PT methods has similar values. The error bar for the 2PT data is estimated from a set of ten runs at each temperature. We find some deviation in the low temperature. At low temperatures, as the dynamics become slow, we need longer runs to get a converged DOS. Figure 15 shows the effect the time step has on the value of total entropy at lower temperatures. With an increase in the time step, the entropy value approaches the value calculated using the TI method. However, at longer times, the slope of the curve decreases.

Configurational entropy, $S_{c}$, obtained in the two different methods is plotted in Fig. 16. We find that the values of Kauzmann temperature $\left(T_{K}\right)$ using two different methods are close, which validates the applicability of the $2 \mathrm{PT}$ method for the calculation of the configurational entropy.

We have compared the density of states calculated from the calculation of Hessian and the Fourier transform of the velocity autocorrelation function. We find that both the methods show a similar result in a density of states (see Fig. 17).

To increase the accuracy of the configurational entropy, we incorporate the anharmonic contribution into the vibrational entropy. ${ }^{43}$ We estimate the anharmonic contribution of the total potential energy as

$$
U_{a n h}(T)=U(T)-U_{I S}-\frac{3}{2} N T,
$$

where $\mathrm{U}(\mathrm{T})$ is the total potential energy at temperature $\mathrm{T}, U_{I S}$ is the corresponding inherent structure energy, and the last term is the harmonic vibrational energy of all the particles. The anharmonic energy can be expanded around temperature $\mathrm{T}=0$ as

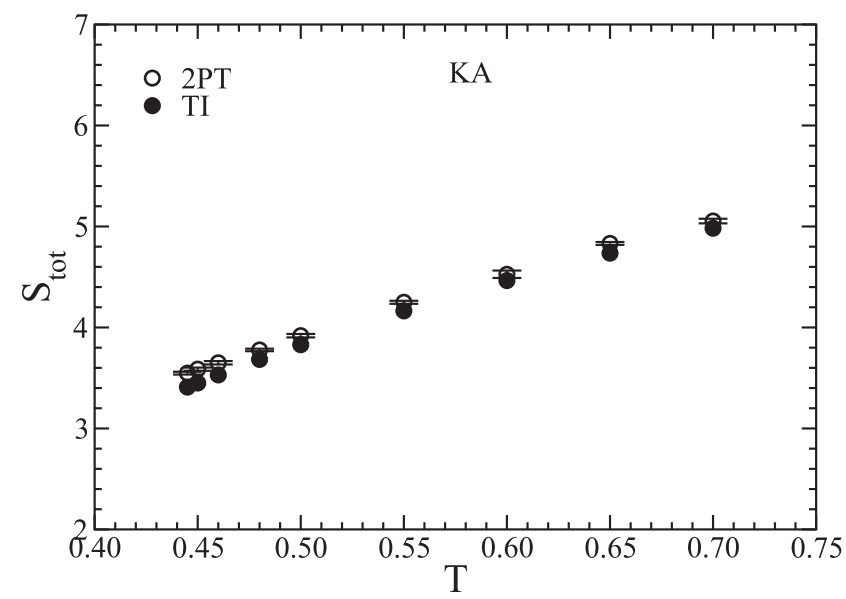

FIG. 14. Stot vsT for the KA model using the TI and the 2PT method. The two methods agree reasonably well. A small systematic deviation in the low temperature regime is due to limited averaging possible for the 2PT method; see Fig. 15.

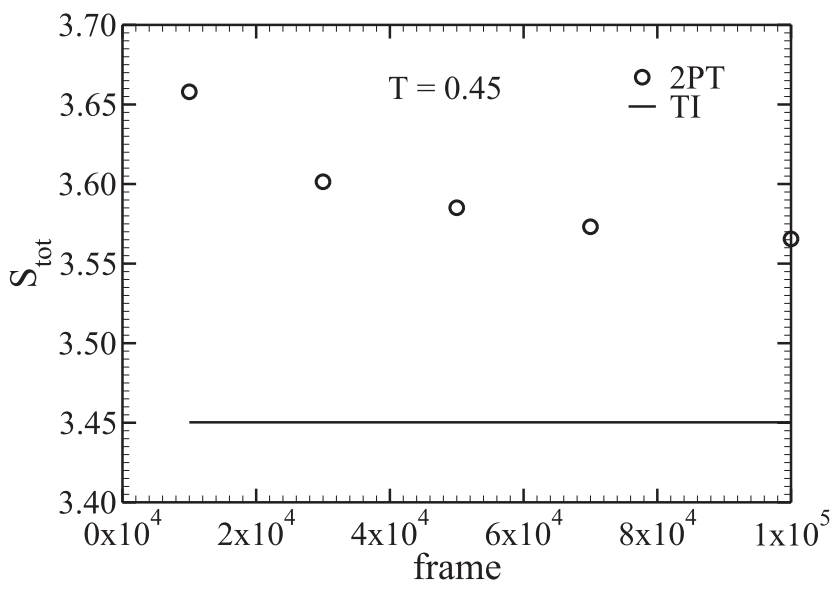

FIG. 15. The total entropy via the 2PT method as a function of the number of time frames over which the velocity autocorrelation function is integrated to obtain the spectral density at a low temperature $T=0.45$. For comparison, we also plot the entropy value obtained using the TI method. The difference decreases with the increasing time interval, but the rate of convergence becomes slower at longer times.

$$
U_{a n h}(T)=\sum_{j=2} C_{j} T^{j}
$$

The coefficients of expansion $C_{j} s$ are temperature independent. As the system is completely harmonic in the low temperature limit, the sum starts from $j=2$. The anharmonic contribution for the vibrational entropy $\left(S_{a n h}\right)$ can be written as

$$
S_{a n h}(T)=\int_{0}^{T} d T^{\prime} \frac{1}{T^{\prime}} \frac{\partial U_{a n h}\left(T^{\prime}\right)}{\partial T^{\prime}} .
$$

If we substitute Eq. (A3) into Eq. (A4), then we get the anharmonic vibrational entropy as

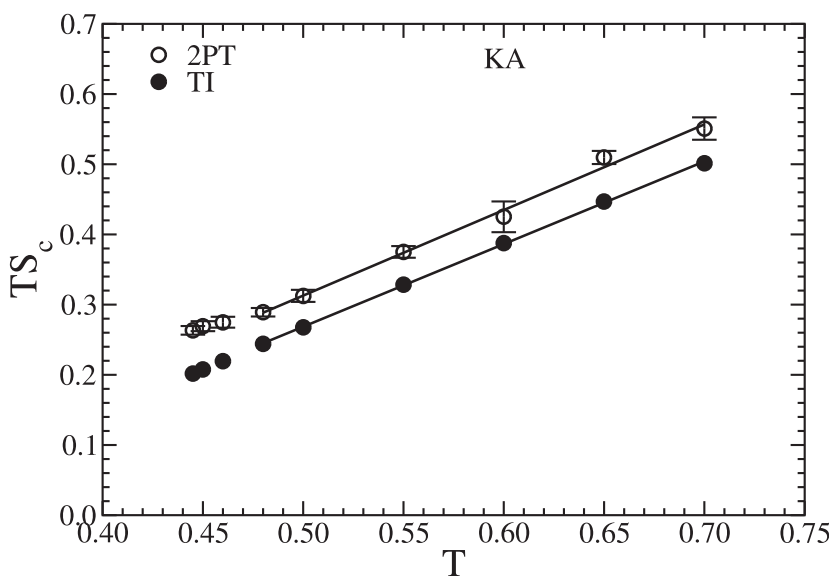

FIG. 16. $T S_{c}$ vs $T$ for the KA model using the $T I$ and the $2 \mathrm{PT}$ methods. The values of $T_{K}$ estimated by the two methods are similar $\left(T_{K}^{T I}=0.27\right.$ and $\left.T_{K}^{2 P T}=0.24\right)$. 


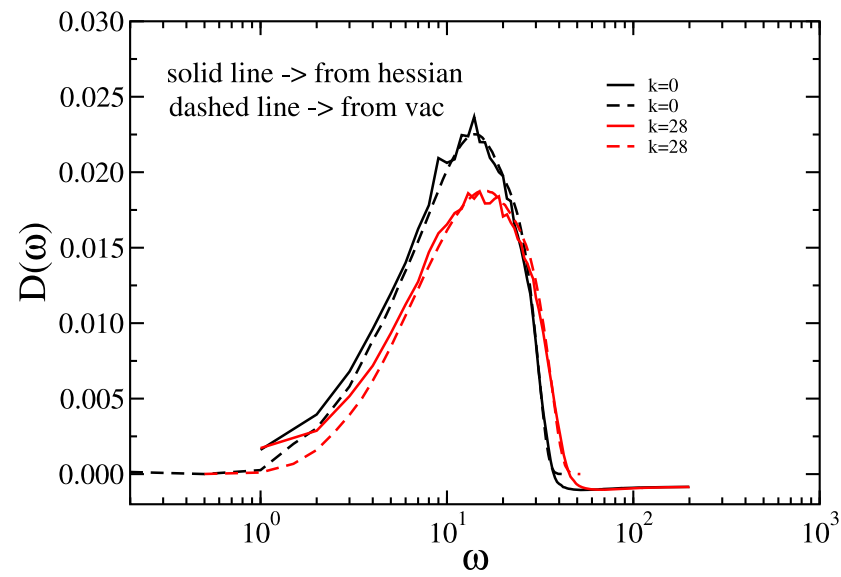

FIG. 17. Density of states calculated from the Hessian and the velocity autocorrelation function for $\mathrm{k}=0$ and $\mathrm{k}=28$ systems. Both the methods show the similar result.

$$
S_{a n h}(T)=\sum_{j=2} \frac{j}{j-1} C_{j} T^{j-1}
$$

In our simulation, we first estimate the anharmonic potential energy, as shown in Fig. 18. We then fit the simulation data to a polynomial function of T [Eq. (A3)] to obtain the coefficients. We use these coefficients to obtain $S_{\text {anh }}$ using Eq. (A5). We find that for both $\mathrm{k}=0$ and $\mathrm{k}=28$ systems, $S_{a n h}$ is negative at all temperatures, thus lowering the vibrational entropy (Fig. 3) and increasing the configurational entropy (Fig. 4).

We also use the Frenkel and Ladd (FL) method to estimate the vibrational entropy of our system. ${ }^{44}$ In the FL method, a perturbed Hamiltonian is introduced,

$$
\beta H(\alpha)=\beta H(0)+\alpha \sum_{i=1}^{N}\left|\mathbf{r}-\mathbf{r}_{0 i}\right|^{2},
$$

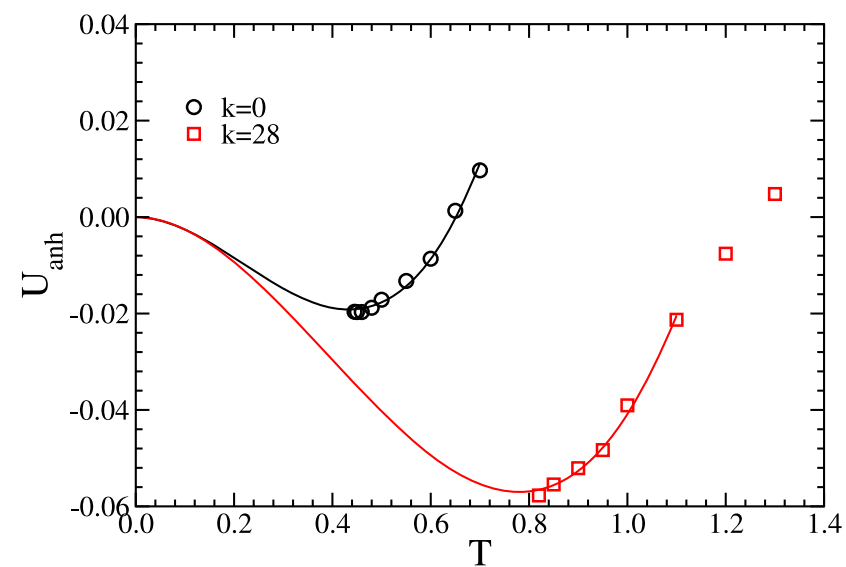

FIG. 18. An harmonic potential energy $\left(U_{\text {anh }}\right)$ vs temperature for $k=0$ and 28 systems. The solid lines are the fitted line extrapolated to the zero temperature value.

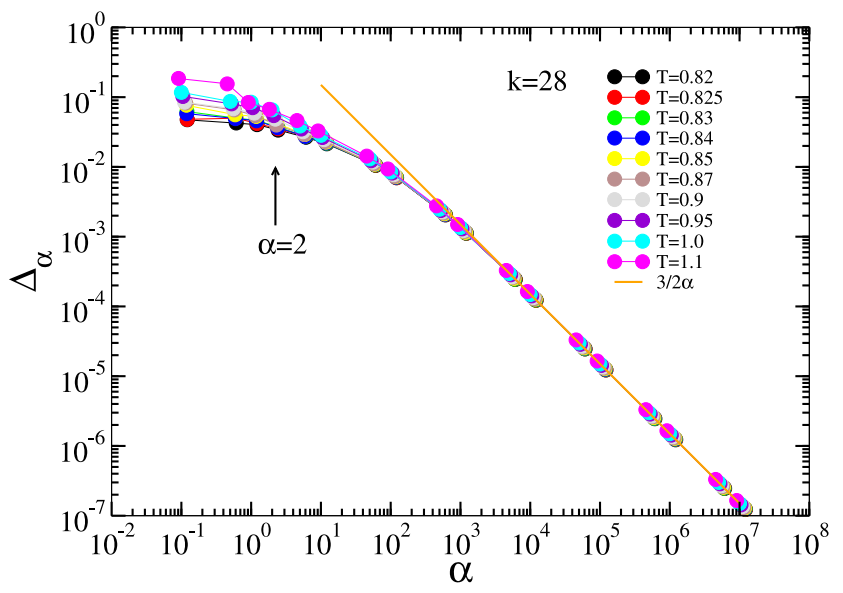

FIG. 19. Mean square displacement $\left(\Delta_{\alpha}\right)$ vs $\alpha$ plot for the $\mathrm{k}=28$ system at different temperatures. The solid line gives the behavior of mean square displacement for the Einstein solid, $\Delta_{\alpha}=3 / 2 \alpha$.

where $H(0)$ is the original unperturbed Hamiltonian of the system, $\beta=\frac{1}{k_{B} T}, \alpha$ is the spring constant which determines the strength of perturbation, and $\mathbf{r}_{0 i}$ is the initial position of the $\mathrm{i}$-th particle of the unperturbed system. By varying $\alpha$, a series of system is obtained, which interpolates between the original system $(\alpha=0)$ and the Einstein crystal (large $\alpha$ values). For each $\alpha$ value, we calculate the mean square displacement (MSD) $\left(\Delta_{\alpha}=\sum_{i=1}^{N}\left\langle\left|\mathbf{r}-\mathbf{r}_{0 i}\right|^{2}\right\rangle / N\right)$ and the vibrational entropy,

$$
S_{F L}=S_{E}\left(\alpha_{\max }\right)+\int_{0}^{\alpha_{\max }} d \alpha \Delta_{\alpha}
$$

The entropy of Einstein solid is $S_{E}\left(\alpha_{\max }\right)=\frac{3}{2}-3 \ln \lambda-\frac{3}{2} \ln \left(\frac{\alpha_{\max }}{\pi}\right)$, where $\lambda$ is the de Broglie wavelength. Figure 19 shows the mean square displacement at different $\alpha$ values for different temperatures.

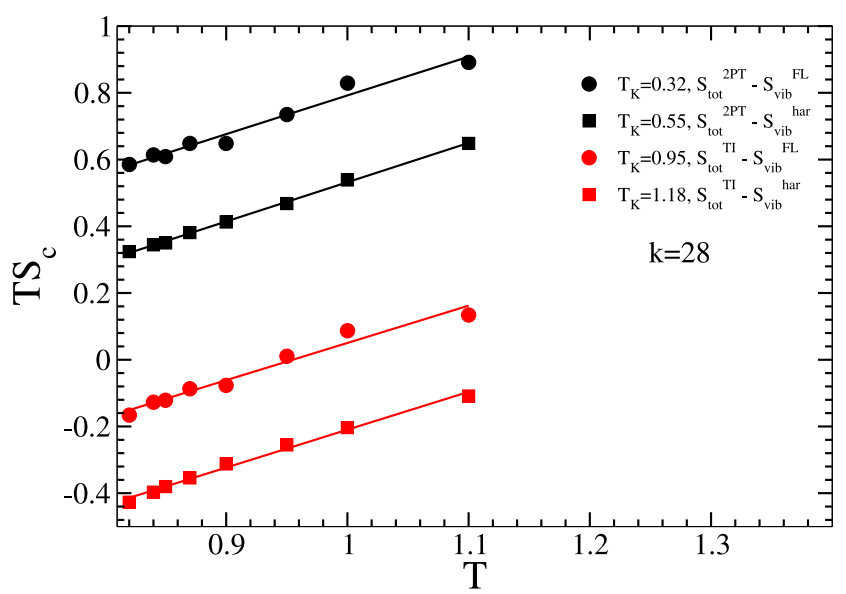

FIG. 20. $T S_{c}$ vs $T$ plot for $\mathrm{TI}$ and $2 \mathrm{PT}$ methods of the $\mathrm{k}=28$ system. The $\mathrm{T}$ method gives negative $S_{c}$ even when the vibrational entropy is calculated from the FL method, $S_{v i b}^{F L}$. The 2PT method does not give such a unphysical entropy value. 
At a high $\alpha$ value, $\Delta_{\alpha}=3 / 2 \alpha$ follows the behavior of an Einstein solid. At small values of $\alpha$, the MSD is not flat, suggesting that eventually, at long time, the particle will escape the cage. Since we are interested in obtaining the vibrational contribution, we choose a lower limit of $\alpha=\alpha_{\min }$ such that the particle remains inside the cage. Thus, to calculate $S_{v i b}$, we integrate Eq. (A7) from $\alpha_{\min }$ to $\alpha_{\max }$. Here, we assume that $\alpha_{\min }=2$ and $\alpha_{\max }=10^{7}$. The contribution from lower values of $\alpha\left(\alpha<\alpha_{\min }\right)$ is assumed to be given by $\Delta_{\alpha_{\min }} \alpha_{\min }$. The vibrational entropy thus obtained is lower than the harmonic approximation and closer to the entropy obtained, including the anharmonic contribution (Fig. 3). Figure 20 shows the $T S_{c}$ vs $\mathrm{T}$ plot for the $\mathrm{k}=28$ system where we calculate $S_{c}$ from both the TI and 2PT methods using vibrational contribution of entropy from the harmonic and FL method. We find that $S_{c}$ becomes negative when we calculate total entropy using the TI method and the 2PT method does not give such negative entropy.

\section{APPENDIX B: PINNED SYSTEM ENTROPY}

In a pinned system, a fraction $c$ of the particles is pinned. The details about the pinned system have been discussed in simulation
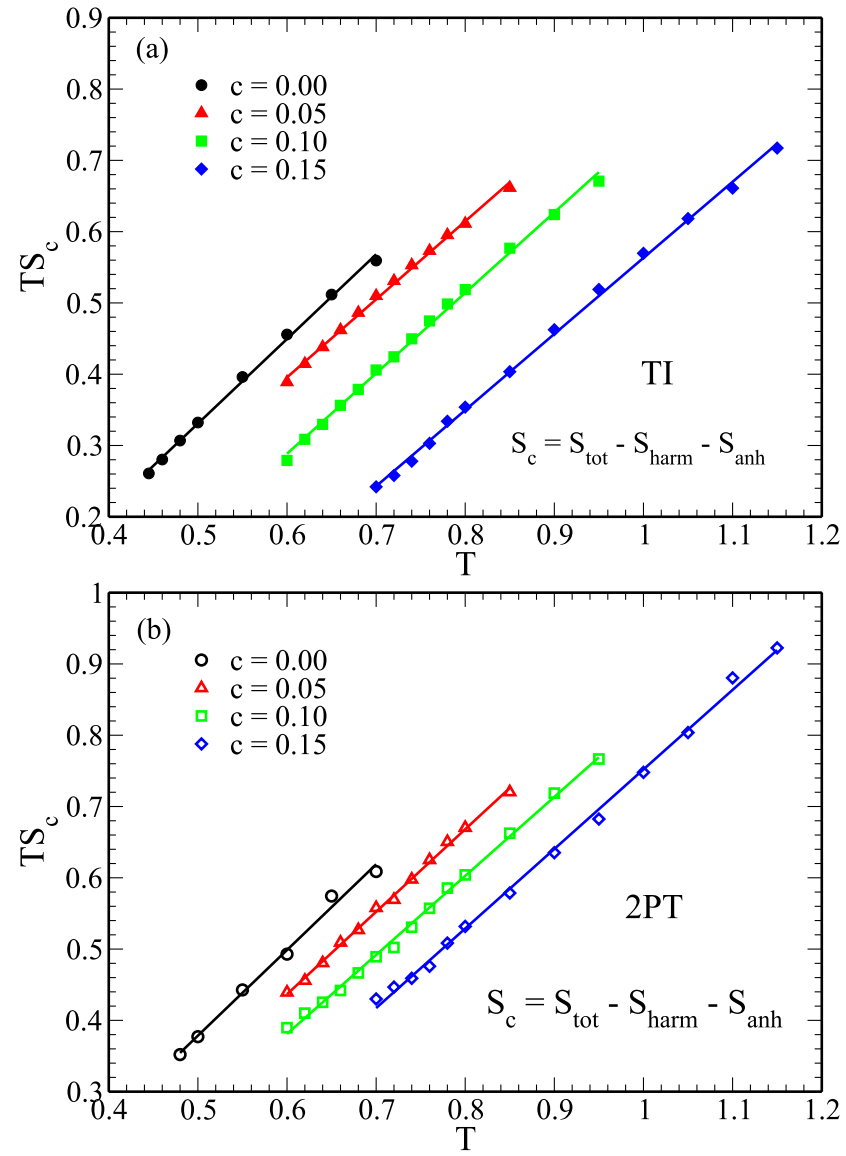

FIG. 21. $T S_{c}$ vs $T$ for $c=0,0.5,0.10$, and 0.15 systems using (a) the TI method and (b) the 2PT method. $S_{c}$ is computed by including the anharmonic contribution. $T_{K}^{T I}$ and $T_{K}^{2 P T}$ increase with increasing pinning concentration, but $T_{K}^{2 P T}<T_{K}^{T I}$; see Table III. details (see Sec. II B). Using the TI method, the total entropy of moving particle in the pinned system, $S_{\text {tot }}$, is given by ${ }^{3}$

$$
\begin{aligned}
S_{\text {tot }}= & \frac{3 M}{2}-\frac{3 M}{2} \ln \left(\frac{2 \pi T}{h^{2}}\right)+M\left(1-\ln \frac{N}{V}\right) \\
& -\sum_{i=1}^{2} N_{i} \ln \frac{N_{i}}{N}+\beta^{*}\langle U\rangle-\int_{0}^{\beta^{*}} d \beta\langle U\rangle,
\end{aligned}
$$

where $N_{1}$ and $N_{2}$ are the number of moving particles of types $A$ and $B$, respectively. $V$ is the total volume of the system and $M$ is the total number of moving particles. The total potential energy of the system is $U=U_{M P}+U_{M M}$, where $U_{M M}$ and $U_{M P}$ denote the interaction energy between moving-moving particles and moving-pinned particles, respectively.

The temperature dependence of the configurational entropy after taking care of the anharmonic contribution is plotted in
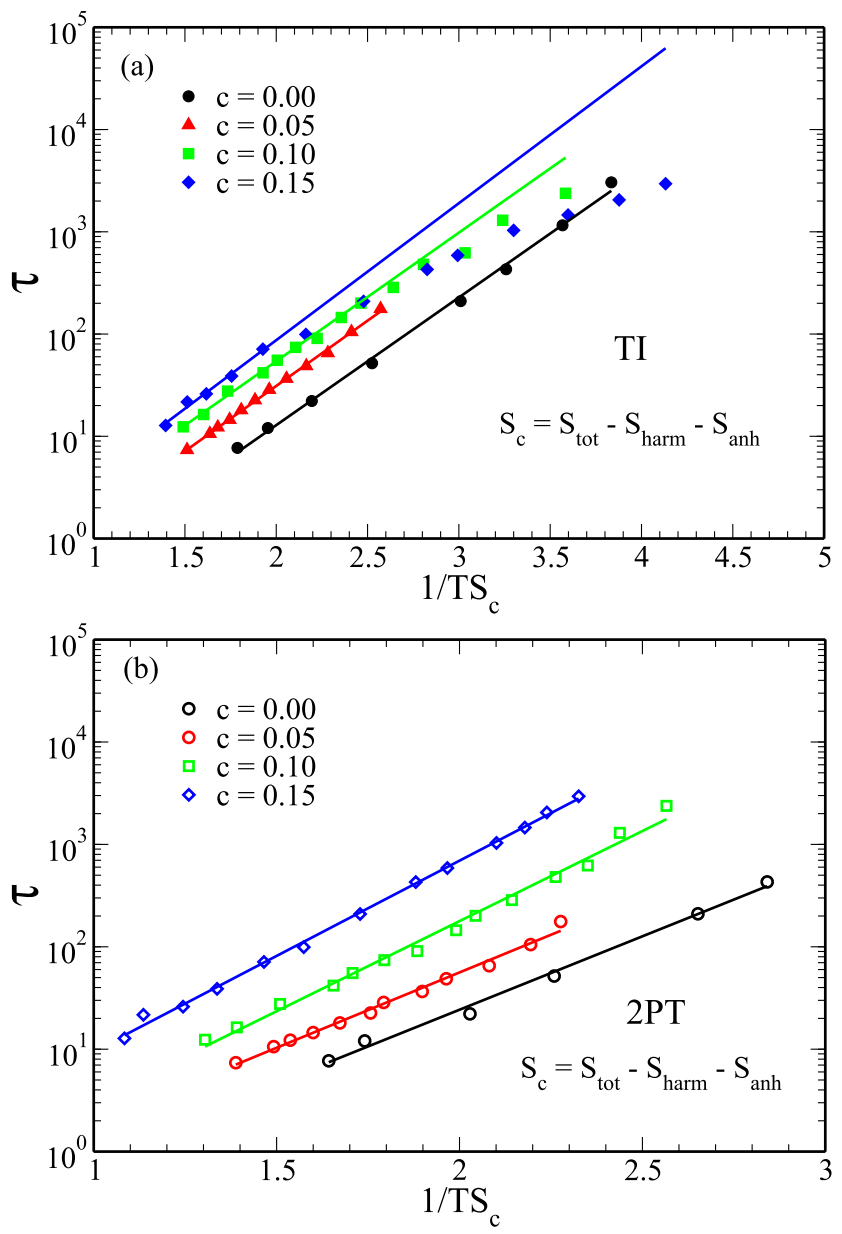

FIG. 22. Testing the AG relation between $\tau$ and $\frac{1}{T S_{c}}$ for $c=0,0.5,0.10$, and 0.15 systems using (a) the TI method and (b) the 2PT method. $S_{c}$ is computed by including the anharmonic contribution. In the temperature range studied here, the $A G$ relation is violated for $c=0.10$ and $c=0.15$ when entropy is calculated using the TI method. However, the AG relation holds when entropy is calculated via the 2PT method. 
TABLE III. The value of all characteristic temperatures for systems with different "c" values. $T_{K}^{T I}\left(\right.$ anh) and $T_{K}^{2 P T}$ (anh) are Kauzmann temperature estimated from $\mathrm{Tl}$ and $2 \mathrm{PT}$, respectively, after the addition of anharmonic contribution.

\begin{tabular}{lcc}
\hline \hline $\mathrm{c}$ & $\mathrm{T}_{K}^{T I}(\mathrm{anh})$ & $\mathrm{T}_{K}^{2 P T}(\mathrm{anh})$ \\
\hline 0.00 & 0.22 & 0.18 \\
0.05 & 0.24 & 0.22 \\
0.10 & 0.34 & 0.26 \\
0.15 & 0.47 & 0.33 \\
\hline \hline
\end{tabular}

Fig. 21(a), and the corresponding Adam-Gibbs plot is shown in Fig. 22(a). Even after the addition of the anharmonic contribution, the AG relationship is violated. In Fig. 21(b), we plot the temperature dependence of the configurational entropy where the total entropy is calculated using the 2PT method and the anharmonic contribution is taken into consideration. We show the $\mathrm{AG}$ plot of the same data in Fig. 22(b). We find that when the total entropy is calculated using the 2PT method, the AG relationship holds, and the temperature where the entropy vanishes is also lower than that given by the TI method (see Table III).

\section{REFERENCES}

${ }^{1}$ S. Sastry, P. G. Debenedetti, and F. H. Stillinger, Nature 393, 554 (1998).

${ }^{2}$ A. Banerjee, M. K. Nandi, S. Sastry, and S. Maitra Bhattacharyya, J. Chem. Phys. 147, 024504 (2017).

${ }^{3}$ P. Patel, M. K. Nandi, U. K. Nandi, and S. Maitra Bhattacharyya, J. Chem. Phys. 154, 034503 (2021).

${ }^{4}$ W. Götze, J. Phys.: Condens. Matter 11, A1 (1999).

${ }^{5}$ W. M. Du et al., Phys. Rev. E 49, 2192 (1994).

${ }^{6}$ P. Lunkenheimer, A. Pimenov, and A. Loidl, Phys. Rev. Lett. 78, 2995 (1997).

${ }^{7}$ K. Kim and S. Saito, J. Chem. Phys. 138, 12 A506 (2013).

${ }^{8}$ W. Kob and H. C. Andersen, Phys. Rev. E 51, 4626 (1995).

${ }^{9}$ E. Flenner and G. Szamel, Phys. Rev. E 72, 031508 (2005).

${ }^{10}$ E. Flenner and G. Szamel, Phys. Rev. E 72, 011205 (2005).

${ }^{11}$ V. Lubchenko and P. G. Wolynes, J. Chem. Phys. 119, 9088 (2003).

${ }^{12}$ T. R. Kirkpatrick and P. G. Wolynes, Phys. Rev. A 35, 3072 (1987).

${ }^{13}$ A. Cavagna, Phys. Rep. 476, 51 (2009).

${ }^{14}$ G. Adam and J. H. Gibbs, J. Chem. Phys. 43, 139 (1965).

${ }^{15}$ W. Kauzmann, Chem. Rev. 43, 219 (1948).

${ }^{16}$ M. Ozawa, C. Scalliet, A. Ninarello, and L. Berthier, J. Chem. Phys. 151, 084504 (2019).

${ }^{17}$ C. A. Angell, J. Res. Natl. Inst. Stand. Technol. 102, 171 (1997).

${ }^{18}$ R. Richert and C. A. Angell, J. Chem. Phys. 108, 9016 (1998).

${ }^{19}$ J. H. Magill, J. Chem. Phys. 47, 2802 (1967).

${ }^{20}$ S. Takahara, O. Yamamuro, and T. Matsuo, J. Phys. Chem. 99, 9589 (1995).
${ }^{21}$ K. L. Ngai, J. Phys. Chem. B 103, 5895 (1999).

${ }^{22}$ C. Alba-Simionesco, C. R. Acad. Sci., Ser. IV: Phys., Astrophys. 2, 203 (2001).

${ }^{23}$ C. M. Roland, S. Capaccioli, M. Lucchesi, and R. Casalini, J. Chem. Phys. 120, 10640 (2004)

${ }^{24}$ D. Cangialosi, A. Alegría, and J. Colmenero, Europhys. Lett. 70, 614 (2005).

${ }^{25}$ E. Masiewicz et al., Sci. Rep. 5, 13998 (2015).

${ }^{26}$ A. D. S. Parmar, S. Sengupta, and S. Sastry, Phys. Rev. Lett. 119, 056001 (2017).

${ }^{27}$ U. K. Nandi, W. Kob, and S. Maitra Bhattacharyya, J. Chem. Phys. 154, 094506 (2021).

${ }^{28}$ R. Mari and J. Kurchan, J. Chem. Phys. 135, 124504 (2011).

${ }^{29}$ S. Sastry, PhysChem Comm 3, 79 (2000).

${ }^{30}$ S.-T. Lin, M. Blanco, and W. A. Goddard, J. Chem. Phys. 119, 11792 (2003).

${ }^{31}$ S.-T. Lin, P. K. Maiti, and W. A. Goddard, J. Phys. Chem. B 114, 8191 (2010).

${ }^{32}$ M. Moid, S. Sastry, C. Dasgupta, T. A. Pascal, and P. K. Maiti, J. Chem. Phys. 154, 164510 (2021).

${ }^{33}$ M. Ozawa, W. Kob, A. Ikeda, and K. Miyazaki, Proc. Natl. Acad. Sci. U. S. A. 112, 6914 (2015).

${ }^{34}$ M. Ozawa, W. Kob, A. Ikeda, and K. Miyazaki, Proc. Natl. Acad. Sci. U. S. A. 112, E4821 (2015).

${ }^{35}$ S. Chakrabarty, S. Karmakar, and C. Dasgupta, Sci. Rep. 5, 12577 (2015).

${ }^{36}$ S. Chakrabarty, S. Karmakar, and C. Dasgupta, Proc. Natl. Acad. Sci. U. S. A. 112, E4819 (2015).

${ }^{37}$ S. Sengupta, F. Vasconcelos, F. Affouard, and S. Sastry, J. Chem. Phys. 135, 194503 (2011).

${ }^{38}$ D. A. McQuarrie, Statistical Mechanics Book (University Science Books, 1975).

${ }^{39}$ P.-K. Lai, C.-M. Hsieh, and S.-T. Lin, Phys. Chem. Chem. Phys. 14, 15206 (2012).

${ }^{40}$ S. Sastry, Nature 409, 164 (2001).

${ }^{41}$ S. Sastry, Phys. Rev. Lett. 85, 590 (2000).

${ }^{42}$ B. Doliwa and A. Heuer, Phys. Rev. Lett. 80, 4915 (1998)

${ }^{43}$ F. Sciortino, J. Stat. Mech.: Theory Exp. 2005, P05015.

${ }^{44}$ D. Frenkel and A. J. C. Ladd, J. Chem. Phys. 81, 3188 (1984).

${ }^{45}$ M. Ozawa, A. Ikeda, K. Miyazaki, and W. Kob, Phys. Rev. Lett. 121, 205501 (2018).

${ }^{46}$ L. Berthier et al., Proc. Natl. Acad. Sci. U. S. A. 114, 11356 (2017)

${ }^{47}$ M. Ozawa and L. Berthier, J. Chem. Phys. 146, 014502 (2017).

${ }^{48}$ Y. Rosenfeld, Phys. Rev. A 15, 2545 (1977).

${ }^{49}$ Y. Rosenfeld, J. Phys.: Condens. Matter 11, 5415 (1999).

${ }^{50}$ M. Singh, M. Agarwal, D. Dhabal, and C. Chakravarty, J. Chem. Phys. 137, 024508 (2012).

${ }^{51}$ A. Banerjee, S. Sengupta, S. Sastry, and S. M. Bhattacharyya, Phys. Rev. Lett. 113, 225701 (2014).

${ }^{\mathbf{5 2}}$ A. Banerjee, M. K. Nandi, and S. M. Bhattacharyya, J. Chem. Sci. 129, 793 (2017).

${ }^{53}$ I. H. Bell, J. C. Dyre, and T. S. Ingebrigtsen, Nat. Commun. 11, 4300 (2020).

${ }^{54}$ S. Chakrabarty, R. Das, S. Karmakar, and C. Dasgupta, J. Chem. Phys. 145, 034507 (2016).

${ }^{55}$ I. Williams et al., J. Phys.: Condens. Matter 30, 094003 (2018).

${ }^{56}$ L. Berthier, M. Ozawa, and C. Scalliet, J. Chem. Phys. 150, 160902 (2019). 